TRANSACTIONS OF THE

AMERICAN MATHEMATICAL SOCIETY

Volume 363, Number 9, September 2011, Pages 4527-455

S 0002-9947(2011)05334-1

Article electronically published on April 19, 2011

\title{
UNIQUE DETERMINATION \\ OF PERIODIC POLYHEDRAL STRUCTURES BY SCATTERED ELECTROMAGNETIC FIELDS
}

\author{
GANG BAO, HAI ZHANG, AND JUN ZOU
}

\begin{abstract}
This work is concerned with the unique determination of a periodic diffraction grating profile in three dimensions by some scattered electromagnetic fields measured above the grating. In general, it is well known that global uniqueness may not be true when the measurement is only taken for one incident field. Our goal is to completely characterize the global uniqueness properties when the periodic structure is of polyhedral type. Corresponding to each incident plane wave, we are able to classify all unidentifiable structures into three classes and show that any periodic polyhedral structure can be uniquely determined by one incident field if and only if it belongs to none of the three classes. Consequently, the minimum number of incident waves required for the unique determination of a periodic polyhedral structure can be easily read.
\end{abstract}

\section{INTRODUCTION}

This work is concerned with the unique determination of a bi-periodic diffraction grating in three dimensions by the scattered electromagnetic fields corresponding to some incident plane waves. A grating structure $S$ is said to be bi-periodic of period $\Lambda=\left(\Lambda_{1}, \Lambda_{2}\right)$ if for any point $x=\left(x_{1}, x_{2}, x_{3}\right) \in S$ the point $\left(x_{1}+n_{1} \Lambda_{1}, x_{2}+\right.$ $\left.n_{2} \Lambda_{2}, x_{3}\right)$ also belongs to $S$ for all integers $n_{1}$ and $n_{2}$. We consider such a periodic grating structure $S$, which is ruled on a perfect conductor. The medium above $S$ is assumed to be homogenous with a constant dielectric coefficient $\epsilon_{0}>0$ and magnetic permeability $\mu_{0}>0$, and the corresponding region is denoted by $\Omega$. Let $E^{i}(x)=s e^{i q \cdot x}$ (with time dependence $e^{-i \omega t}$ ) be an incident time-harmonic electromagnetic wave incident to the grating structure $S$ from above. Vectors $s$ and $q$ are orthogonal, and the incident direction $q$ can be written as $q=\left(\alpha_{1}, \alpha_{2},-\beta\right)$ for some $\beta>0$. Accordingly, the wave number $k$ and frequency $\omega$ involved are respectively given by

$$
k=|q|, \quad \omega=|q| / \sqrt{\epsilon_{0} \mu_{0}} .
$$

Received by the editors April 30, 2009.

2010 Mathematics Subject Classification. Primary 35R30, 35Q61, 78A45.

Key words and phrases. Periodic structure, inverse scattering, uniqueness, dihedral group.

The first author was supported in part by the NSF grants DMS-0604790, DMS-0908325, CCF0830161, EAR-0724527, and DMS-0968360, and by the ONR grant N00014-02-1-0365.

The third author was substantially supported by Hong Kong RGC grants (Projects 404606 and 404407) and partially supported by the Cheung Kong Scholars Programme through Wuhan University. 
For the subsequent analysis, we will frequently need vector $\alpha=\left(\alpha_{1}, \alpha_{2}, 0\right)$, partially taken from vector $q$.

Let $E$ be the total field, which is the sum of the incident field and the scattered field. Then $E$ satisfies the following vector-valued Helmholtz system:

$$
\begin{array}{rll}
\Delta E+k^{2} E & =0 & \text { in } \Omega, \\
\operatorname{div} E & =0 & \text { in } \Omega, \\
\nu \times E & =0 & \text { on } S,
\end{array}
$$

where $\nu$ is the unit outward normal vector to the surface $S$.

In view of the bi-periodic structure of $S$, we are only interested in the quasiperiodic solutions $E$ to the system (11)-(3), i.e., $e^{-i \alpha \cdot x} E$ is periodic respectively with period $\Lambda_{1}$ in the $x_{1}$ direction and $\Lambda_{2}$ in the $x_{2}$ direction; see 8 , 12. We also impose a radiation condition in the $x_{3}$ direction by assuming that $E$ is composed of bounded outgoing plane waves plus the incident wave $E^{i}$. Then it follows from the knowledge of the fundamental solution to the periodic Helmholtz equation (cf. 8], [12]) that $E$ can be expressed in the following form:

$$
E(x)=E^{i}(x)+\sum_{n \in \mathbf{Z}^{2}} A^{n} e^{i q^{n} \cdot x} \text { for all } x \text { in } \mathbf{R}^{3} \text { above the highest point on } S,
$$

where all $A^{n}$ 's are complex vectors, called the Rayleigh coefficients, and all $q^{n}$ 's are given by $q^{n}=\alpha^{n}+\alpha+\left(0,0, \beta^{n}\right)$ with $\alpha^{n}=\left(2 \pi n_{1} / \Lambda_{1}, 2 \pi n_{2} / \Lambda_{2}, 0\right)$ and

$$
\beta^{n}=\left\{\begin{aligned}
\sqrt{k^{2}-\left|\alpha^{n}+\alpha\right|^{2}}, & k^{2} \geq\left|\alpha^{n}+\alpha\right|^{2}, \\
i \sqrt{-k^{2}+\left|\alpha^{n}+\alpha\right|^{2}}, & k^{2}<\left|\alpha^{n}+\alpha\right|^{2} .
\end{aligned}\right.
$$

One can see from (5) that there are only finitely many $n$ 's for which $\beta^{n}$ are real, i.e., there are only a finite number of propagating plane waves in the scattered field while the remaining modes decay exponentially along the $x_{3}$ direction.

Throughout this work we assume that

$$
k^{2} \neq\left|\alpha^{n}+\alpha\right|^{2} \quad \text { for all } n \in \mathbf{Z}^{2} .
$$

The assumption (6) is the usual condition to ensure the uniqueness of the forward scattering problem (11)-(4) (cf. [8, 12]).

For the sake of convenience, we introduce the index set

$$
\Xi=\left\{n \in \mathbf{Z}^{2} ; \beta^{n}>0\right\}
$$

and denote by $E_{p}$ the propagating field, namely, the part of the total field $E$ in (4) with those exponentially decaying modes removed:

$$
E_{p}(x)=E^{i}(x)+\sum_{n \in \Xi} A^{n} e^{i q^{n} \cdot x} .
$$

Unlike the total field $E$ in the expression (44), the complex vector-valued function $E_{p}$ can be extended to the whole space $\mathbf{R}^{3}$ naturally, and this fact will be used repeatedly in the subsequent analysis.

Given the periodic structure $S$ and the incident field $E^{i}$, the forward diffraction problem is to solve for the system (10)-(4) the total field $E$. The direct diffraction problem has been well studied mathematically; see, e.g., [12, [4- 6]. The current work is mainly concerned with an inverse problem associated with the system (11)(41). For a given incident wave $E^{i}$, assume that the total field $E$ can be measured on a plane $\Gamma_{b}=\left\{x_{3}=b\right\}$ above the structure $S$. Then we want to find out how many incident waves should be sent so that the measurements of the resulting total fields 
on the plane $\Gamma_{b}$ can uniquely determine the shape and position of the structure $S$. It is well known that global uniqueness with one incident wave is generally not true. This can be seen from a simple example that when one incident wave is sent, two grating profiles, both parallel to the plane $\left\{x_{3}=0\right\}$ with distance of a certain multiple of the wavelength of the incident wave, generate the same total field $E$ in the domain above $\Gamma_{b}$. As far as the general periodic grating profiles are concerned, the global uniqueness of this inverse problem still remains open. However, when it is confined to some special classes of periodic structures, important progress has been made in recent years, particularly in the two-dimensional case. Hettlich and Kirsch showed in [19] that a finite number of incident waves are sufficient to identify a $C^{2}$-smooth periodic structure in two dimensions. In a series of works by Elschner, Schmidt and Yamamoto (see [14, 15] and [18), the global uniqueness problem for the class of periodic polygonal structures was studied and the minimal number of incident waves to ensure the global uniqueness was obtained. On the other hand, Bao and Friedman established in [7] the local stability for gratings that consist of pairs of smooth periodic curves, while the case of interfaces with corner points was addressed by Elschner and Schmidt in [16].

To our knowledge, there are only two uniqueness results for the aforementioned inverse problem in the three-dimensional case, both for gratings which can be described by graphs of $C^{2} \Lambda$-periodic functions. Local uniqueness was established in [8], where a crucial step was to estimate a lower bound of the first eigenvalue to the problem (11) in a convex domain. The global uniqueness result was obtained in [3] with one incident plane wave when the medium above the perfect conductor was assumed to be lossy, i.e., $k$ had a non-zero imaginary part, while for the case when $k$ was real, global uniqueness was established with infinitely many incident waves.

In this work we will restrict ourselves to the Lipschitz polyhedral gratings, so the grating structure $S$ is assumed to be a Lipschitz polyhedral surface with a period $\Lambda$. For each given incident wave, we are able to identify three classes of polyhedral gratings and demonstrate that these are all the possible polyhedral gratings which cannot be uniquely determined by the incident wave. Using these results, we can determine the minimum number of incident waves to uniquely determine a polyhedral grating. The novelty of our approach lies in the following two significant aspects:

- Our central analysis is basically constructive, which provides clear characterizations of all unidentifiable periodic grating structures, even though we adopt contradicting arguments from time to time. It should be noted that all the methodologies used in the existing uniqueness studies for inverse obstacle scattering problems are carried out purely by contradiction arguments; see [2], 8], [9, 10, [11, [17, 18, 19], 21], 23] and the references therein.

- A major technique used in this work is dihedral group theory, which is for the first time introduced in the uniqueness study for inverse scattering problems and turns out to be extremely helpful to characterize the unidentifiable periodic structures.

The rest of the paper is organized as follows. In Section 2 we present some technical tools and observations which are frequently needed in the subsequent analysis. In Section 3, we discuss how to start from one or two perfect planes of the total field to find all the possible grating profiles to which the global uniqueness 
fails. Finally, the main result is established on the uniqueness of the scattering problem in Section 4

\section{Preliminaries}

We begin by introducing the following conventions and notation:

(1) For any vector $b \in \mathbf{R}^{3}$, its norm is denoted by $\|b\|$. For convenience, we may often view a point $r \in \mathbf{R}^{3}$ also as the vector originating from the origin which directs to the point $r$.

(2) A vector $r \in \mathbf{R}^{3}$ is said to be parallel to a line $l$ in $\mathbf{R}^{3}$ with a tangential unit vector $\nu$ if $r \| \nu$. For a plane $\Pi$ in $\mathbf{R}^{3}$, we denote by $\nu_{\Pi}$ the unit normal vector to $\Pi$. A vector $r$ is said to be parallel to a plane $\Pi$ in $\mathbf{R}^{3}$ if $r \perp \nu_{\Pi}$.

(3) For any $c \in \mathbf{C}^{3}$ and $r \in \mathbf{R}^{3}$, the dot product $c \cdot r=0$ means $\operatorname{Re}(c) \cdot r=0$ and $\operatorname{Im}(c) \cdot r=0$. The same conventions will be made for the relations $c \| r$, $c \times r$ and $c \perp \Pi$ for any plane $\Pi$ in $\mathbf{R}^{3}$.

(4) Let $\Pi$ be a plane in $\mathbf{R}^{3}$; we denote by $R_{\Pi}$ the reflection with respect to the plane $\Pi$ in $\mathbf{R}^{3}$. The reflection $R_{\Pi}$ is always understood to act on a point in $\mathbf{R}^{3}$.

Let $\Pi^{\prime}$ be the plane that passes through the origin and is parallel to $\Pi$, and let $R_{\Pi}^{\prime}$ be the derivative of $R_{\Pi}$, namely the linear part of $R_{\Pi}$. One can see that $R_{\Pi}^{\prime}$ is the reflection with respect to the plane $\Pi^{\prime}$. For a point $r \in \mathbf{R}^{3}, R_{\Pi}^{\prime} r$ can also be viewed as the reflection of the vector that initiates from the origin and points to the point $r$, with respect to the plane $\Pi^{\prime}$. By natural extension, we apply $R_{\Pi}^{\prime}$ to complex vectors in $\mathbf{C}^{3}$ as well.

(5) For a set $\mathcal{A}$, we denote by $|\mathcal{A}|$ the number of elements in $\mathcal{A}$.

(6) Let $G$ be a group which acts on a set $\mathcal{A}$, and let $a \in \mathcal{A}$. Then $G\{a\}$ means the orbit of $a$ under the action of the group $G$. By the group property, we know that for any two elements $a, b \in \mathcal{A}$, either $G\{a\}=G\{b\}$ or $G\{a\} \cap$ $G\{b\}=\emptyset$.

Next we introduce two concepts, i.e., perfect sets and perfect planes, that are crucial to the subsequent analysis.

Definition 2.1. Let $F: O \rightarrow \mathbf{C}^{3}$ be a given analytic complex vector-valued function in a domain $O \subset \mathbf{R}^{3}$. $\mathcal{P}_{F}$ is called the perfect set of $F$ if

$$
\begin{array}{r}
\mathcal{P}_{F}=\left\{x \in O ; \nu \times\left. F\right|_{\Pi \cap B_{r}(x) \cap O}=0 \text { for some } r>0\right. \\
\text { and plane } \Pi \text { passing through } x\} .
\end{array}
$$

The points in $\mathcal{P}_{F}$ are called perfect points of $F$. For any $x \in \mathcal{P}_{F}$, we denote by $\Pi$ the plane involved in the definition of $\mathcal{P}_{F}$. Furthermore, we let $\widetilde{\Pi}$ be the connected component of $\Pi \cap O$ containing $x$. Then by the analyticity of $F$ and analytic continuation, we have $\nu \times F=0$ on $\widetilde{\Pi}$. In the sequel, such $\widetilde{\Pi}$ will be referred to as a perfect plane of $F$. We also use $\mathcal{P}_{F}$ to denote the set of perfect planes of $F$ whenever there is no cause for confusion.

Note that the electric field $E$ to the system (11)-(3) is analytic in the domain $\Omega$, which leads us to the important reflection property of a perfect plane in the following lemma; see 22.

Lemma 2.1 (Reflection Principle). Let $O$ be a domain in $\mathbf{R}^{3}$ which is symmetric with respect to a plane $\Pi$, and let $E$ be an electric field in $O$ satisfying the vectorvalued Helmholtz equations (11)-(2). Assume that $\tilde{\Pi}$ is a connected open subset in 
$\Pi \cap O$. Then $\tilde{\Pi}$ is a perfect plane of $E$ if and only if the following relation holds:

$$
E(x)+R_{\Pi}^{\prime}\left(E\left(R_{\Pi}(x)\right)\right)=0 \text { in } O .
$$

Moreover, if $\Gamma \subset O$ or $\Gamma \subset \partial O$ is a perfect plane of $E$, then $R_{\Pi}(\Gamma)$ is also a perfect plane of $E$.

The following two lemmas present some results that will be used repeatedly in the subsequent sections.

Lemma 2.2. Let $n \in \mathbf{N}$, let $\lambda_{1}, \lambda_{2}, \ldots, \lambda_{n}$ be $n$ real numbers, and assume that the following relation holds for $n$ complex vectors $a_{1}, \ldots, a_{n} \in \mathbf{C}^{m}$ for $m \in \mathbf{N}$ :

$$
\lim _{t \rightarrow \infty} \sum_{j=1}^{n} a_{j} e^{i \lambda_{j} t}=0 .
$$

Then

$$
\sum_{j=1}^{n} a_{j} e^{i \lambda_{j} t}=0 \quad \forall t \in \mathbf{R} .
$$

Moreover, if $\lambda_{1}, \lambda_{2}, \ldots, \lambda_{n}$ are distinct, then $a_{1}=a_{2}=\cdots=a_{n}=0$.

Proof. We prove the second part of the lemma first by induction. The case $n=1$ is trivial. Now we assume that the conclusion holds for some $n \in \mathbf{N}$; we need to show that it also holds for $n+1$. Assume that there exists $n+1$ distinct real numbers $\lambda_{1}, \lambda_{2}, \ldots, \lambda_{n+1}$ and $n+1$ complex vectors $a_{1}, \ldots, a_{n+1}$ such that

$$
\lim _{t \rightarrow \infty} \sum_{j=1}^{n+1} a_{j} e^{i \lambda_{j} t}=0 .
$$

If there is some $1 \leq j \leq n+1$ such that $a_{j}=0$, then the result for $n+1$ follows from the assumption for $n$. Now assume that $a_{j} \neq 0$ for $1 \leq j \leq n+1$. Then we can derive the following relations by multiplying both sides of (10) respectively by $e^{i \lambda_{1}}$ and $e^{i \sqrt{2} \lambda_{1}}$ :

$$
\begin{aligned}
\lim _{t \rightarrow \infty} \sum_{j=1}^{n+1} a_{j} e^{i \lambda_{1}} e^{i \lambda_{j} t} & =0, \\
\lim _{t \rightarrow \infty} \sum_{j=1}^{n+1} a_{j} e^{i \sqrt{2} \lambda_{1}} e^{i \lambda_{j} t} & =0,
\end{aligned}
$$

and the following relations by changing the variable $t$ in (10) respectively into $t+1$ and $t+\sqrt{2}$ :

$$
\begin{aligned}
\lim _{t \rightarrow \infty} \sum_{j=1}^{n+1} a_{j} e^{i \lambda_{j}} e^{i \lambda_{j} t} & =0, \\
\lim _{t \rightarrow \infty} \sum_{j=1}^{n+1} a_{j} e^{i \sqrt{2} \lambda_{j}} e^{i \lambda_{j} t} & =0 .
\end{aligned}
$$

Subtracting (13) from (11) gives

$$
\lim _{t \rightarrow \infty} \sum_{j=2}^{n+1} a_{j}\left(e^{i \lambda_{1}}-e^{i \lambda_{j}}\right) e^{i \lambda_{j} t}=0,
$$


which along with the assumption for $n$ yields

$$
a_{j}\left(e^{i \lambda_{1}}-e^{i \lambda_{j}}\right)=0 \quad \text { for } 2 \leq j \leq n+1 .
$$

Similarly, we deduce from (12) and (14) that

$$
a_{j}\left(e^{i \sqrt{2} \lambda_{1}}-e^{i \sqrt{2} \lambda_{j}}\right)=0 \quad \text { for } 2 \leq j \leq n+1 .
$$

Since $e^{i \lambda_{1}}-e^{i \lambda_{j}}$ and $e^{i \sqrt{2} \lambda_{1}}-e^{i \sqrt{2} \lambda_{j}}$ cannot be zero simultaneously, we conclude that $a_{j}=0$ for $2 \leq j \leq n+1$, which is a contradiction. This completes the induction and the proof of the second part of the lemma.

To prove the first part, for each $\lambda_{j}$ we combine all the terms in (9) which have the same $\lambda_{j}$, and then we apply the previously proved result for the resulting expression.

Lemma 2.3. For any $n, m \in \mathbf{N}$, let $q_{1}, q_{2}, \ldots, q_{n}$ be $n$ vectors in $\mathbf{R}^{3}$, and let $a_{1}, a_{2}$, $\ldots, a_{n}$ be $n$ vectors in $\mathbf{C}^{m}$ such that

$$
\sum_{j=1}^{n} a_{j} e^{i q_{j} \cdot x}=0 \quad \text { for all } x \in \mathbf{R}^{3} .
$$

Then it holds that

$$
\sum_{i: q_{i}=q_{j}} a_{i}=0 \text { for each } j .
$$

If $q_{1}, q_{2}, \ldots, q_{n}$ are all different from each other, then $a_{1}=a_{2}=\cdots=a_{n}=0$.

Proof. It is easy to see that the first result of the lemma follows directly from the second one, so we shall show only the second result. First we prove that for any $q \in \mathbf{R}^{3}$ and $q \neq 0$,

$$
\lim _{r \rightarrow+\infty} \frac{1}{r^{3}} \iiint_{B(r)} e^{i q \cdot x} d x=0,
$$

where $B(r)=\left\{x \in \mathbf{R}^{3} ;\|x\| \leq r\right\}$. Indeed, let $R$ be a rotation in $\mathbf{R}^{3}$ such that $R^{T} q=(\|q\|, 0,0)$. Then we can write

$$
\begin{aligned}
\iiint_{B(r)} e^{i q \cdot x} d x & =\iiint_{B(r)} e^{i q \cdot R x} d R x=\iiint_{B(r)} e^{i R^{T} q \cdot x} d x \\
& =\iiint_{B(r)} e^{i\|q\| x_{1}} d x \\
& =\iint_{x_{2}^{2}+x_{3}^{2} \leq r^{2}} d x_{2} d x_{3} \int_{-\sqrt{r^{2}-x_{2}^{2}-x_{3}^{2}}}^{\sqrt{r^{2}-x_{2}^{2}-x_{3}^{2}}} e^{i\|q\| x_{1}} d x_{1} .
\end{aligned}
$$

Note that there exists a constant $C=C(\|q\|)$ such that

$$
\left|\int_{t_{1}}^{t_{2}} e^{i\|q\| x_{1}} d x_{1}\right| \leq C(\|q\|) \quad \forall t_{1}, t_{2} \in \mathbf{R},
$$

so we derive

$$
\left|\iiint_{B(r)} e^{i q \cdot x} d x\right| \leq C(\|q\|) 4 \pi r^{2},
$$

from which the equality (15) follows immediately. 
Now, we let $a_{1}, \ldots, a_{n} \in \mathbf{C}^{3}$ be such that

$$
\sum_{j=1}^{n} a_{j} e^{i q_{j} \cdot x}=0
$$

If there is some $1 \leq l \leq n$ such that $a_{l} \neq 0$, then we get from (16) that

$$
a_{l}=-\sum_{j \neq l} a_{j} e^{i\left(q_{j}-q_{l}\right) \cdot x} .
$$

Now integrating both sides of (17) over the ball $B(r)$ and then dividing by $r^{3}$ yields that $a_{l}=0$ by means of (15), leading to a contradiction. This completes the proof of Lemma 2.3

\section{Classification of Unidentifiable Periodic Structures}

The aim of this section is to determine and classify all the unidentifiable periodic structures corresponding to a given incident field.

3.1. Observations and auxiliary tools. We start by recalling some basic notation from Section 1

$E^{i}(x)=s e^{i q \cdot x}$ : the incident electric field;

$E(x)$ : the total field;

$S$ : the grating profile of bi-period $\Lambda$;

$\Gamma_{b}$ : the plane $\left\{x_{3}=b\right\}$ above the grating $S$, on which the total field $E$ is measured;

$\Omega_{b}$ : the domain above the plane $\Gamma_{b}$;

$\Xi=\left\{n \in \mathbf{Z}^{2} ; \beta^{n}>0\right\}$.

We also write:

$$
\begin{aligned}
& \Xi_{0}=\left\{n \in \Xi ; A^{n} \neq 0\right\}, \quad \mathcal{Q}=\{q\} \cup\left\{q^{n}\right\}_{n \in \Xi_{0}}, \\
& E_{p}(x)=s e^{i q \cdot x}+\sum_{n \in \Xi_{0}} A^{n} e^{i q^{n} \cdot x}, \\
& \mathcal{P}=\left\{\Pi ; \Pi \text { is a perfect plane of } E_{p}\right\} .
\end{aligned}
$$

We now introduce a very useful concept to the subsequent analysis.

Definition 3.1. For a given plane $\Pi$ in $\mathbf{R}^{3}$, a periodic part $S_{l}$ of the entire grating structure $S$ is called a $\Pi$-reflecting periodic part if each face of $S_{l}$ can be reflected by $\Pi$ into the domain $\Omega_{b}$.

The following lemma presents a crucial observation.

Lemma 3.1. If $\Pi$ is a perfect plane of the total field $E$, then $\Pi$ is also a perfect plane of the propagating field $E_{p}$.

Proof. We separate the proof into two cases: $\Pi \nVdash\left\{x_{3}=0\right\}$ and $\Pi \|\left\{x_{3}=0\right\}$.

Case $1 . \Pi$ is not parallel to the plane $\left\{x_{3}=0\right\}$. We choose $x_{0} \in \Pi$ and two linearly independent vectors $v_{1}, v_{2}$ in $\mathbf{R}^{3}$ such that their components in the $x_{3}$ direction are positive and that $x_{0}+\lambda v_{1}+\mu v_{2}$ belongs to $\Pi$ for all $\lambda>0, \mu>0$. Note that for $x_{3} \geq b$ we have the expansion

$$
E(x)=E^{i}(x)+\sum_{n \in \mathbf{Z}^{2}} A^{n} e^{i q^{n} \cdot x}:=E_{p}(x)+E_{s}(x),
$$


which, along with the property that $E_{s}$ decays exponentially as $x_{3} \rightarrow \infty$ and $E \times \nu_{\Pi}=0$ on $\Pi$ by the definition of a perfect plane, yield that

$$
\lim _{\lambda \rightarrow \infty} \nu_{\Pi} \times E_{p}\left(x_{0}+\lambda v_{1}+\mu v_{2}\right)=0 \text { for all } \mu>0,
$$

i.e.,

$$
\lim _{\lambda \rightarrow \infty}\left\{s e^{i q \cdot\left(x_{0}+\lambda v_{1}+\mu v_{2}\right)}+\sum_{n \in \Xi} A^{n} e^{i q^{n} \cdot\left(x_{0}+\lambda v_{1}+\mu v_{2}\right)}\right\} \times \nu_{\Pi}=0 \text { for all } \mu>0 .
$$

Then it follows from Lemma 2.2 that

$$
\left\{s e^{i q \cdot\left(x_{0}+\lambda v_{1}+\mu v_{2}\right)}+\sum_{n \in \Xi} A^{n} e^{i q^{n} \cdot\left(x_{0}+\lambda v_{1}+\mu v_{2}\right)}\right\} \times \nu_{\Pi}=0 \quad \forall \mu>0, \lambda \in \mathbf{R} .
$$

Considering that all exponential functions involved above are analytic, we know immediately that the above equality holds for all $\mu \in \mathbf{R}$ by analytic continuation. So the desired result is proved for Case 1.

Case 2. $\Pi$ is parallel to the plane $\left\{x_{3}=0\right\}$. Let $\Pi=\left\{x_{3}=c\right\}$ with $c>b$. Since $\Pi$ is a perfect plane of $E$, we have for all $x \in \Pi$ that

$$
\begin{aligned}
e^{-i \alpha \cdot x} E(x) \times e_{3} & =e^{-i \alpha \cdot x}\left\{E^{i}(x)+\sum_{n \in \mathbf{Z}^{2}} A^{n} e^{i q^{n} \cdot x}\right\} \times e_{3} \\
& =\left(s e^{-\beta c}+A^{0} e^{\beta c}\right) \times e_{3}+\sum_{n \in \mathbf{Z}^{2} \backslash\{0\}} A^{n} e^{i \beta^{n} c} \times e_{3} e^{i \alpha^{n} \cdot x} \\
& =0 .
\end{aligned}
$$

Note that $\left\{e^{i \alpha^{n} \cdot x}\right\}_{n \in \mathbf{Z}^{2}}$ is an orthogonal family in $L^{2}\left(\left(0, \Lambda_{1}\right) \times\left(0, \Lambda_{2}\right)\right)$ of variables $x_{1}$ and $x_{2}$. Therefore we obtain

$$
\left(s e^{-\beta c}+A^{0} e^{\beta c}\right) \times e_{3}=0, \quad A^{n} e^{i \beta^{n} c} \times e_{3}=0 \quad \forall n \in \mathbf{Z}^{2} \backslash\{0\} .
$$

Then for all $x \in \Pi$ we derive

$$
e^{-i \alpha x} E_{p}(x) \times \nu_{\Pi}=\left(s e^{-\beta c}+A^{0} e^{\beta c}\right) \times e_{3}+\sum_{n \in \Xi} A^{n} e^{i \beta^{n} c} \times e_{3} e^{i \alpha^{n} \cdot x}=0,
$$

so we have $E_{p}(x) \times \nu_{\Pi}=0$ for all $x \in \Pi$. This completes the proof of Case 2 .

The subsequent analysis is mainly based on the study of the perfect planes of the total field $E$. But we recall that a perfect plane of $E$ is usually not a true plane in $\mathbf{R}^{3}$. By means of Lemma 3.1, it would be much more convenient for us to investigate the perfect planes of the propagating field $E_{p}$, as those perfect planes are truly two-dimensional planes in $\mathbf{R}^{3}$. To see this, we may first observe that $E_{p}$ is analytic in the whole space $\mathbf{R}^{3}$ since $E_{p}$ contains only a finite number of exponential functions, and each of the functions is analytic in $\mathbf{R}^{3}$. Then by the analytic continuation, each perfect plane of $E_{p}$, namely each element in $\mathcal{P}$, is a truly two-dimensional plane.

For each incident field $E^{i}$, we will find all the grating structures which cannot be identified by the incident field. We begin with the following assumption, which is the first fundamental fact to be established in the proof of our main result on the global uniqueness in Section 4.

Assumption 1. There exists a perfect plane of $E$, denoted by $\tilde{\Pi}_{0}$, such that $\tilde{\Pi}_{0} \cap$ $\Omega_{b} \neq \emptyset$ and $\Pi_{0}$ is not perpendicular to the plane $\left\{x_{3}=0\right\}$. 
Next we present four important observations. The first one follows from the definition of the set $\mathcal{Q}$ in (18), while the second follows directly from the Reflection Principle (Lemma 2.1) and Lemma 3.1.

Proposition 1. Each of the vectors in $\mathcal{Q}$ except $q$ has a positive $x_{3}$ component.

Proposition 2. For each $\Pi_{0}$-reflecting periodic part $S_{l}$ of grating $S$, each face of $S_{l}$ can be reflected with respect to $\Pi_{0}$ to a perfect plane in $\mathcal{P}$.

Proposition 3. For each perfect plane $\Pi$ in $\mathcal{P}$, we have $R_{\Pi}^{\prime} \mathcal{Q}=\mathcal{Q}$ and $R_{\Pi} \mathcal{P}=\mathcal{P}$. Moreover, both maps $R_{\Pi}^{\prime}: \mathcal{Q} \rightarrow \mathcal{Q}$ and $R_{\Pi}: \mathcal{P} \rightarrow \mathcal{P}$ are bijective.

Proof. By the Reflection Principle (Lemma 2.1) the following relation holds for each perfect plane $\Pi \in \mathcal{P}$ :

$$
E_{p}(x)+R_{\Pi}^{\prime}\left(E_{p}\left(R_{\Pi} x\right)\right)=0 \quad \forall x \in \mathbf{R}^{3},
$$

which implies by the definition of $E_{p}$ that

$$
s e^{i q \cdot x}+\sum_{n \in \Xi_{0}} A^{n} e^{i q^{n} \cdot x}+R_{\Pi}^{\prime} s e^{i q \cdot R_{\Pi} x}+\sum_{n \in \Xi_{0}} R_{\Pi}^{\prime} A^{n} e^{i q^{n} \cdot R_{\Pi} x}=0 \quad \forall x \in \mathbf{R}^{3} .
$$

It is easy to check that $R_{\Pi} x=R_{\Pi}^{\prime} x+R_{\Pi} 0$ and that $R_{\Pi}^{\prime}$ is symmetric, so we can rewrite the above equation as

$$
\begin{aligned}
& s e^{i q \cdot x}+\sum_{n \in \Xi_{0}} A^{n} e^{i q^{n} \cdot x}+\left(R_{\Pi}^{\prime} s e^{i q \cdot R_{\Pi} 0}\right) e^{i R_{\Pi}^{\prime} q \cdot x} \\
& +\sum_{n \in \Xi_{0}}\left(R_{\Pi}^{\prime} A^{n} e^{i q^{n} \cdot R_{\Pi} 0}\right) e^{i R_{\Pi}^{\prime} q^{n} \cdot x}=0 \quad \forall x \in \mathbf{R}^{3} .
\end{aligned}
$$

From this relation and Lemma 2.3. we know immediately that each vector of $\mathcal{Q}$ must be reflected by $R_{\Pi}^{\prime}$ to one and only one vector in $\mathcal{Q}$. Thus $R_{\Pi}^{\prime} \mathcal{Q} \subseteq \mathcal{Q}$. By noting that $R_{\Pi}^{\prime}$ is a bijective transformation in $\mathbf{R}^{3}$, we know that $R_{\Pi}^{\prime} \mathcal{Q}=\mathcal{Q}$ and the map $R_{\Pi}^{\prime}: \mathcal{Q} \rightarrow \mathcal{Q}$ is also a bijection.

The remaining results in Proposition 3 about $\mathcal{P}$ follow directly from the Reflection Principle (Lemma 2.1) and the bijectiveness of $R_{\Pi}$ in $\mathbf{R}^{3}$.

Proposition 4. For a $\Pi_{0}$-reflecting periodic part $S_{l}$ of grating $S$, each face of $S_{l}$ must lie on some perfect plane in $\mathcal{P}$.

Proof. By Proposition 2 we have $R_{\Pi_{0}} S_{l} \subset \mathcal{P}$. On the other hand, it follows from Lemma 3.1 that $\Pi_{0} \in \mathcal{P}$. Hence we know $S_{l}=R_{\Pi_{0}}\left(R_{\Pi_{0}} S_{l}\right) \subset \mathcal{P}$ by Proposition 3 .

The following lemma presents some useful properties about perfect planes.

Lemma 3.2. Let $F=t e^{i p \cdot x}$ be one of the Fourier modes of $E_{p}$ in (19). Therefore,

(a) if $\Pi$ is a perfect plane of the field $F=t e^{i p \cdot x}$, then $t \perp \Pi$;

(b) if $\Pi$ is a perfect plane in $\mathcal{P}$ such that $R_{\Pi}^{\prime} p=p$, then $\Pi$ is also a perfect plane of the field $F$;

(c) if $\Pi$ and $\Pi^{*}$ are two perfect planes in $\mathcal{P}$ such that $R_{\Pi}^{\prime} p=R_{\Pi^{*}}^{\prime} p$, then $\Pi \| \Pi^{*}$.

Proof. (a) is a direct consequence of the definition of a perfect plane. To see (b), we apply Lemma 2.3 to equality (21) with $\Pi$ to obtain

$$
t e^{i p \cdot x}+R_{\Pi}^{\prime} t e^{i p \cdot R_{\Pi} x}=0
$$


which shows that the field $F=t e^{i p \cdot x}$ satisfies the symmetric relation (8) as in Lemma 2.1 with respect to the plane $\Pi$, so $\Pi$ is a perfect plane of $F$.

Finally, we prove (c). We do it in two cases: $R_{\Pi_{1}}^{\prime} p=R_{\Pi^{*}}^{\prime} p=p$ or $R_{\Pi^{\prime}}^{\prime} p=R_{\Pi^{*}}^{\prime} p \neq$ $p$. The assertion for the former case follows readily from (a) and (b), while the proof of the latter follows from the fact that $\nu_{\Pi}\left\|\left(p-R_{\Pi^{\prime}}^{\prime} p\right)\right\| \nu_{\Pi^{*}}$.

3.2. First class of unidentifiable gratings. We are now ready to start the process to find all of the unidentifiable gratings corresponding to the incident field $E^{i}=s e^{i q \cdot x}$. More precisely, these structures are classified into three classes. The first unidentifiable class corresponds to the special case when all the planes in $\mathcal{P}$ are parallel to $\Pi_{0}$, as stated in the following lemma.

Lemma 3.3. If all the planes in $\mathcal{P}$ are parallel to $\Pi_{0}$, then both $\Pi_{0}$ and $S$ are parallel to $\left\{x_{3}=0\right\}$, and the distance between any two neighboring perfect planes in $\mathcal{P}$ is the same, equal to $\pi / \beta$.

On the other hand, if $\Pi$ is a plane that is parallel to $\Pi_{0}$ and the distance between $\Pi$ and $\Pi_{0}$ is some integer multiple of $\pi / \beta$, then $\Pi$ is a perfect plane in $\mathcal{P}$.

Proof. By Proposition 4 we know that each face of any $\Pi_{0}$-reflecting periodic part $S_{l}$ of $S$ can be reflected by $\Pi_{0}$ into a plane in $\mathcal{P}$, so they are parallel to the plane $\Pi_{0}$. Due to the periodic structure of $S$, all the faces of $S$ must be parallel to $\Pi_{0}$ as well. But this is possible only if $S$ is an entire plane in $\mathbf{R}^{3}$. Noting that $S$ is bounded in the $x_{3}$ direction, both $S$ and $\Pi_{0}$ are parallel to the plane $\left\{x_{3}=0\right\}$.

To see that the distance between any two neighboring perfect planes in $\mathcal{P}$ is the same, let $\Pi$ be a plane in $\mathcal{P}$; then $\Pi\left\|\Pi_{0}\right\|\left\{x_{3}=0\right\}$. Let $q^{*}=R_{\Pi}^{\prime} q$; then $q^{*} \neq q$ since otherwise $q\|\Pi\|\left\{x_{3}=0\right\}$. By Proposition 3, we have $\left\{q, q^{*}\right\} \subset \mathcal{Q}$. We now claim that $\mathcal{Q}=\left\{q, q^{*}\right\}$. If it is not true, there is some $\tilde{q} \in \mathcal{Q}$ such that $\tilde{q} \neq q, q^{*}$. By Proposition 3. $R_{\Pi}^{\prime} \tilde{q} \in \mathcal{Q}$. As $\Pi \|\left\{x_{3}=0\right\}$, either $\tilde{q}$ or $R_{\Pi}^{\prime} \tilde{q}$ has a non-positive $x_{3}$ component, which is in contradiction to Proposition 10

Now, we have $q^{*}=R_{\Pi_{0}}^{\prime} q$ and $\mathcal{Q}=\left\{q, q^{*}\right\}$. One can see easily that $q^{*}=$ $\left(\alpha_{1}, \alpha_{2}, \beta\right)=q^{(0,0)}$. We further write the propagating field $E_{p}$ in (19) as $E_{p}=$ $s e^{i q \cdot x}+A^{*} e^{i q^{*} \cdot x}$. Applying equality (21) respectively to the planes $\Pi_{0}$ and $\Pi$, we get

$$
\begin{aligned}
s+R_{\Pi_{0}}^{\prime} A^{*} e^{i q^{*} \cdot R_{\Pi_{0}} 0} & =0, \\
s+R_{\Pi}^{\prime} A^{*} e^{i q^{*} \cdot R_{\Pi} 0} & =0 .
\end{aligned}
$$

From equation (22), we see that $A^{*}=e^{i q^{*} \cdot R_{\Pi_{0}} 0} R_{\Pi_{0}}^{\prime} s$. Thus $E_{p}$ is totally determined by the incident field $E^{i}$ and the plane $\Pi_{0}$. Since $\Pi \| \Pi_{0}, R_{\Pi_{0}}^{\prime} A^{*}=R_{\Pi}^{\prime} A^{*}$, equation (22) minus equation (23) yields

$$
R_{\Pi}^{\prime} A^{*}\left(e^{i q^{*} \cdot R_{\Pi_{0}} 0}-e^{i q^{*} \cdot R_{\Pi} 0}\right)=0,
$$

which implies

$$
e^{i q^{*} \cdot\left(R_{\Pi_{0}} 0-R_{\Pi} 0\right)}=1 .
$$

Let dist $\left(\Pi, \Pi_{0}\right)$ be the distance between the planes $\Pi_{0}$ and $\Pi$; then we have $R_{\Pi_{0}} 0-$ $R_{\Pi} 0= \pm 2 \operatorname{dist}\left(\Pi, \Pi_{0}\right) e_{3}$. By substituting this equality into equation (24) and using the fact that $q^{*} \cdot e_{3}=-q \cdot e_{3}=\beta$, we get $\operatorname{dist}\left(\Pi, \Pi_{0}\right)=\frac{m \pi}{\beta}$ for some integer $m$.

Finally, let $\Pi$ be a plane in $\mathbf{R}^{3}$ that is parallel to $\Pi_{0}$ with a distance of some integer multiple of $\pi / \beta$ from $\Pi_{0}$. Then one can easily deduce using the above derivations that the relation (21) holds for $\Pi$, and therefore $\Pi$ is also a perfect 
plane in $\mathcal{P}$ by the Reflection Principle (Lemma 2.1). This completes the proof of Lemma 3.3

Let $r$ be an arbitrary point in $\mathbf{R}^{3}$. Then Lemma 3.3 leads us to the first class of unidentifiable gratings corresponding to the incident field $E^{i}(x)=s e^{i q \cdot x}$ :

$$
\begin{aligned}
\mathcal{S}_{0}(q, r)= & \left\{\text { all planes which are parallel to }\left\{x_{3}=0\right\}\right. \text { and have equal distance } \\
& \pi / \beta \text { between each other, with } r \text { lying on one of the planes }\}
\end{aligned}
$$

Using the class $\mathcal{S}_{0}(q, r)$, we can conclude that any two gratings belonging to the class $\mathcal{S}_{0}(q, r)$ cannot be distinguished by the incident wave $E^{i}$. To see this, let $S_{1}$ and $S_{2}$ be two planes in $\mathcal{S}_{0}(q, r)$. Then $E_{p}$, which is completely determined by two quantities $s$ and $q$ from $E^{i}$ by the proof of Lemma 3.3 is the solution to the system (11)-(4) with the boundary $S$ replaced respectively by $S_{1}$ and $S_{2}$. Therefore two different planes $S_{1}$ and $S_{2}$ correspond to the same total field generated by the incident wave $E^{i}$ in the domain above $S_{1}$ and $S_{2}$.

3.3. Preparation for finding other classes of unidentifiable gratings. We considered the special case in Subsection 3.2 where all perfect planes are parallel to the same plane $\Pi_{0}$. We now proceed to study the more general case when there is some plane in $\mathcal{P}$ which is not parallel to $\Pi_{0}$. As we shall see, this case leads to two other classes of unidentifiable gratings. For this purpose, let $\Pi_{1}$ be a plane in $\mathcal{P}$ which is not parallel to the plane $\Pi_{0}$. We introduce the following notation:

$L$ : the line of intersection between $\Pi_{0}$ and $\Pi_{1}$;

$r$ : an arbitrary but fixed point on $L$;

$\nu$ : a unit tangential vector along $L$, with non-negative $x_{3}$ component;

$\Gamma$ : the plane in $\mathbf{R}^{3}$ which passes through the origin and has normal $\nu$. For convenience, we assign the following orientation to the plane $\Gamma$ : the normal $\nu$ and $\Gamma$ form a right-handed coordinate system in $\mathbf{R}^{3}$.

$P_{\Gamma}$ : the projection from $\mathbf{R}^{3}$ onto $\Gamma$.

$T_{\theta}$ : the rotation on the plane $\Gamma$ about the origin by angle $\theta$. Clearly $T_{\theta}$ can also be viewed as a rotation in the whole space $\mathbf{R}^{3}$ about the axis fixed to be a line passing through the origin and parallel to direction $\nu$. In both cases, the rotation is understood to be counterclockwise with respect to the assigned orientation on the plane $\Gamma$.

We remark that $\nu \nVdash e_{3}$. Otherwise one gets $e_{3} \| \Pi_{0}$ by noting that $\nu \| \Pi_{0}$, contradicting the assumption that $\Pi_{0}$ is not perpendicular to the plane $\left\{x_{3}=0\right\}$. As a result, $\Gamma$ does not coincide with the plane $\left\{x_{3}=0\right\}$.

Viewing $(\nu, \Gamma)$ as a coordinate system, we can split all vectors in $\mathcal{Q}$ as follows:

$$
q=\tau \nu+P_{\Gamma} q, \quad q^{n}=\tau_{n} \nu+P_{\Gamma} q^{n} \text { for } n \in \Xi_{0},
$$

where $\tau$ and $\tau_{n}$ are constants. It is important to observe that

Lemma 3.4. For any $p \in \mathcal{Q}, P_{\Gamma} p \neq 0$.

Proof. The lemma may be proved by contradiction. If $P_{\Gamma} p=0$, then $p$ is parallel to both $\Pi_{0}$ and $\Pi_{1}$; thus $p=R_{\Pi_{0}}^{\prime} p$ and $p=R_{\Pi_{1}}^{\prime} p$. By Lemma 3.2 (c), we know that $\Pi_{0} \| \Pi_{1}$, which contradicts the choice of $\Pi_{0}$ and $\Pi_{1}$. 
Now we define

$$
\begin{aligned}
\mathcal{P}_{0} & =\left\{\Pi ; \Pi \text { is a perfect plane of } E_{p} \text { and passes through the line } L\right\}, \\
G & =\text { the group generated by the reflections }\left\{R_{\Pi}^{\prime} ; \Pi \in \mathcal{P}_{0}\right\} .
\end{aligned}
$$

Lemma 3.5. The following properties are valid for the set $\mathcal{P}_{0}$ and group $G$ :

(1) The number of perfect planes in $\mathcal{P}_{0}$ is finite; in fact, $\left|\mathcal{P}_{0}\right| \leq|\mathcal{Q}|$.

(2) $G$ consists of $\left|\mathcal{P}_{0}\right|$ reflections and $\left|\mathcal{P}_{0}\right|$ rotations, so it has the structure of a dihedral group of order $2\left|\mathcal{P}_{0}\right|$. Also, the angles formed by any two neighboring planes in $\mathcal{P}_{0}$ are all equal.

Proof. To see (1), we take any two planes $\Pi^{*}$ and $\Pi^{* *}$ in $\mathcal{P}_{0}$. One can easily derive by using (25) and $P_{\Gamma} q \neq 0$ (Lemma 3.4) that the following three relations are equivalent:

(a) $R_{\Pi^{*}}^{\prime} q=R_{\Pi^{* *}}^{\prime} q$; (b) $R_{\Pi^{*}}^{\prime}\left(P_{\Gamma} q\right)=R_{\Pi^{* *}}^{\prime}\left(P_{\Gamma} q\right)$; (c) $\Pi^{*}=\Pi^{* *}$. Thus $\left|\left\{R_{\Pi}^{\prime} q ; \Pi \in \mathcal{P}_{0}\right\}\right|=\left|\mathcal{P}_{0}\right|$. Moreover, we have $\left\{R_{\Pi}^{\prime} q ; \Pi \in \mathcal{P}_{0}\right\} \subseteq \mathcal{Q}$ by Proposition 3 , hence $\left|\mathcal{P}_{0}\right| \leq|\mathcal{Q}|<+\infty$.

To show (2), let $\Pi^{*}$ and $\Pi^{* *}$ be two planes in $\mathcal{P}_{0}$ such that the angle, say $\theta$, formed by $\Pi^{*}$ and $\Pi^{* *}$ is the smallest among all the angles formed by any two planes in $\mathcal{P}_{0}$. Then we have $\theta \leq \frac{\pi}{\left|\mathcal{P}_{0}\right|}$. By Proposition 3 we see that $R_{\Pi} \mathcal{P}_{0}=\mathcal{P}_{0}$ for $\Pi=\Pi^{*}$ or $\Pi^{* *}$. Thus all the planes generated by rotating the plane $\Pi^{*}$ about the axis $L$ with angles of integral multiples of $\theta$ belong to $\mathcal{P}_{0}$. We can get at least $\left|\mathcal{P}_{0}\right|$ such planes since $\theta \leq \frac{\pi}{\left|\mathcal{P}_{0}\right|}$. From this we can conclude that all the planes in $\mathcal{P}_{0}$ can be generated in this manner and that the angles formed by any two neighboring planes in $\mathcal{P}_{0}$ are all equal. Then it is easy to check that $G$ consists of $\left|\mathcal{P}_{0}\right|$ reflections and $\left|\mathcal{P}_{0}\right|$ rotations; hence $G$ has the structure of a dihedral group of order $2\left|\mathcal{P}_{0}\right|$.

In the sequel, we denote by $G^{*}$ the subgroup of $G$ which consists of all its rotations. Clearly, we know $\left|G^{*}\right|=\left|\mathcal{P}_{0}\right|$. Note that the identity element, denoted by $I d$, of both the group $G$ and $G^{*}$ is the rotation by the angle $2 \pi$. When the domain which the transformations in $G$ act on is restricted to the plane $\Gamma, G$ reduces to the dihedral group (see [20, e.g.) which acts only on the vectors lying on the plane $\Gamma$.

Next, we present additional properties about the group $G$ and the set $\mathcal{P}_{0}$.

Lemma 3.6. The following properties hold for the group $G$ and the set $\mathcal{P}_{0}$ :

(1) For any $T \in G, T \mathcal{Q}=\mathcal{Q}$ and $T \nu=\nu$; for any $q^{n} \in G\{q\}, \tau_{n}=\tau$.

(2) $\left|\left\{R_{\Pi}^{\prime} q ; \Pi \in \mathcal{P}_{0}\right\}\right|=\left|G^{*}\{q\}\right|=\left|\mathcal{P}_{0}\right|$.

(3) $|G\{q\}|=2\left|\mathcal{P}_{0}\right|$ or $|G\{q\}|=\left|\mathcal{P}_{0}\right|$. If $|G\{q\}|=2\left|\mathcal{P}_{0}\right|$, there exists some $q^{1} \in G\{q\}$ such that $G\{q\}=G^{*}\{q\} \cup G^{*}\left\{q^{1}\right\}$. If $|G\{q\}|=\left|\mathcal{P}_{0}\right|$, then $G\{q\}=G^{*}\{q\}$ and there exists a plane $\Pi$ in $\mathcal{P}_{0}$ such that $R_{\Pi}^{\prime} q=q$.

(4) $|G\{q\}| \geq 2$, and there exists at least one element in $G\left\{P_{\Gamma} q\right\}$ whose $x_{3}$ component is non-positive. Furthermore, if $|G\{q\}| \geq 4$, then there are at least two elements in $G\left\{P_{\Gamma} q\right\}$, that have non-positive $x_{3}$ components.

Proof. To see (1), noting the fact that $\Pi \| \nu$ for all $\Pi \in \mathcal{P}_{0}$, we have $R_{\Pi}^{\prime} \nu=\nu$. On the other hand, it follows from Proposition 3 that $R_{\Pi}^{\prime} \mathcal{Q}=\mathcal{Q}$ for all $\Pi \in \mathcal{P}_{0}$. Then by the definition of the group $G$, we know $T \mathcal{Q}=\mathcal{Q}$ and $T \nu=\nu$ for all $T \in G$. To see $\tau_{n}=\tau$ for any $q^{n} \in G\{q\}$, we have $G\{q\}=G\left\{\tau \nu+P_{\Gamma} q\right\}=\tau \nu+G\left\{P_{\Gamma} q\right\}$ by the decomposition (25). Since all the elements in $G\left\{P_{\Gamma} q\right\}$ lie on the plane $\Gamma$, the desired result follows immediately. 
Next we consider (2). We note that $R_{\Pi}^{\prime} q=R_{\Pi^{*}}^{\prime} q$ if and only if $\Pi=\Pi^{*}$ for any two given planes $\Pi$ and $\Pi^{*}$ in $\mathcal{P}_{0}$. Therefore we have $\left|\left\{R_{\Pi}^{\prime} q ; \Pi \in \mathcal{P}_{0}\right\}\right|=\left|\mathcal{P}_{0}\right|$. In addition, we know $P_{\Gamma} q \neq 0$ by Lemma 3.4. so we derive $\left|G^{*}\{q\}\right|=\left|G^{*}\left\{P_{\Gamma} q\right\}\right|=$ $\left|G^{*}\right|=\left|\mathcal{P}_{0}\right|$.

To show (3), we consider the stabilizer subgroup $G_{q}$ of $q$, i.e., $G_{q}=\{T \in$ $G ; T q=q\}$. It follows that $P_{\Gamma} q \neq 0$ by Lemma 3.4 so we know that the only rotation in $G$ which maps $q$ into itself is the identity. On the other hand, there is at most one reflection in $G$ which transforms $q$ into itself. Hence we know $G_{q}=\{I d\}$ or $G_{q}=\left\{I d, R_{\Pi}^{\prime}\right\}$ for some $\Pi \in \mathcal{P}_{0}$; then $\left|G_{q}\right|=1$ or $\left|G_{q}\right|=2$. By the orbitstabilizer theorem and Lagrange's theorem (see [20, e.g.), $|G\{q\}|=\frac{|G|}{\left|G_{q}\right|}$, which implies $|G\{q\}|=2\left|\mathcal{P}_{0}\right|$ or $|G\{q\}|=\left|\mathcal{P}_{0}\right|$.

If $|G\{q\}|=\left|\mathcal{P}_{0}\right|$, we see from the previous analysis that $\left|G_{q}\right|=2$. Hence there is a reflection in $G$ which maps $q$ into itself. That is, there exists a plane $\Pi$ in $\mathcal{P}_{0}$ such that $R_{\Pi}^{\prime} q=q$. For the case with $|G\{q\}|=2\left|\mathcal{P}_{0}\right|=2\left|G^{*}\right|$, we first note from the group's property that either $G^{*}\left\{q^{*}\right\}=G^{*}\{q\}$ or $G^{*}\left\{q^{*}\right\} \cap G^{*}\{q\}=\emptyset$ for all $q^{*} \in G\{q\}$. Therefore there exists some $q^{1} \in G\{q\} \backslash G^{*}\{q\}$. Observing that $\left|G^{*}\{q\}\right|+\left|G^{*}\left\{q^{1}\right\}\right|=2\left|\mathcal{P}_{0}\right|=|G\{q\}|$, we have $G\{q\}=G^{*}\{q\} \cup G^{*}\left\{q^{1}\right\}$.

Finally we consider (4). Clearly it follows from (3) that $|G\{q\}| \geq\left|\mathcal{P}_{0}\right| \geq 2$. On the other hand, we can easily verify that the sum of the vectors in $G^{*}\left\{P_{\Gamma} q\right\}$ is zero, as the set $G^{*}\left\{P_{\Gamma} q\right\}$ is exactly formed by the vertices of a regular $n$-sided polygon ( $\left.n=\left|G^{*}\left\{P_{\Gamma} q\right\}\right|\right)$ centered at the origin. Therefore there exists at least one element in $G^{*}\left\{P_{\Gamma} q\right\} \subset G\left\{P_{\Gamma} q\right\}$ whose $x_{3}$ component is non-positive.

We are left with the case when $|G\{q\}| \geq 4$. Clearly we have $\left|G^{*}\{q\}\right| \geq 4$ or $\left|G^{*}\{q\}\right|=2$. For the former case, there exist at least two elements in $G^{*}\left\{P_{\Gamma} q\right\} \subseteq$ $G\left\{P_{\Gamma} q\right\}$ which have a non-positive $x_{3}$ component, by again using the fact that the set $G^{*}\left\{P_{\Gamma} q\right\}$ consists of the vertices of a regular polygon centered at the origin. For the case with $\left|G^{*}\{q\}\right|=2$, we know that $|G\{q\}|=4$. Then it follows from the previously proved result (3) that

$$
G\left\{P_{\Gamma} q\right\}=G^{*}\left\{P_{\Gamma} q\right\} \cup G^{*}\left\{P_{\Gamma} q^{1}\right\} .
$$

In either of the sets $G^{*}\left\{P_{\Gamma} q\right\}$ and $G^{*}\left\{P_{\Gamma} q^{1}\right\}$, one can find at least one element whose $x_{3}$ component is non-positive. This completes the proof of Lemma 3.6 .

Recalling that the line $L$ is the intersection line between $\Pi_{0}$ and $\Pi_{1}, L$ is either parallel to the plane $\left\{x_{3}=0\right\}$ or unparallel. When $L$ is parallel to the plane $\left\{x_{3}=0\right\}$, the following result holds.

Lemma 3.7. If $L \|\left\{x_{3}=0\right\}$, then $\mathcal{Q}=G\{q\}$, and $\left|\mathcal{P}_{0}\right| \in\{2,3\}$.

Proof. To see $\mathcal{Q}=G\{q\}$, it suffices to show that $\mathcal{Q} \backslash G\{q\}=\emptyset$. If this is not true, there is some $q^{m} \in \mathcal{Q} \backslash G\{q\}$. Then we can write $G\left\{q^{m}\right\}=\tau_{m} \nu+G\left\{P_{\Gamma} q^{m}\right\}$. By Lemma 3.6. at least one element from $G\left\{P_{\Gamma} q^{m}\right\}$ has a non-positive $x_{3}$ component. So does at least one element from $G\left\{q^{m}\right\}$ by noting that the $x_{3}$ component of $\nu$ is zero, leading to a contradiction with Proposition 1. Therefore we have $\mathcal{Q}=G\{q\}$.

As the $x_{3}$ component of $\nu$ is zero, we see from the decomposition (25) that the sign of the $x_{3}$ components of the vectors in $G\{q\}$ are determined by those in $G\left\{P_{\Gamma} q\right\}$. If $\left|\mathcal{P}_{0}\right|>3$, then $|G\{q\}| \geq\left|\mathcal{P}_{0}\right|>3$. Thus there are at least two elements in $G\left\{P_{\Gamma} q\right\}$ whose $x_{3}$ components are non-positive by Lemma 3.6)(3); hence the same for $G\{q\}$. This contradicts Proposition 1, So we have proved that $\left|\mathcal{P}_{0}\right| \in\{2,3\}$. 
Based on Lemma 3.7 our analysis may be decomposed into two cases: $L \|\left\{x_{3}=\right.$ $0\}$ or $L \nVdash\left\{x_{3}=0\right\}$. The former is considered in Subsections 3.5 and 3.6. while the latter is studied in Subsection 3.7. As we shall see, the former case leads us to two classes of grating structures which cannot be identified by the incident field $E^{i}$, while the latter case will be shown to be impossible to occur.

3.4. A simple transformation. In the subsequent analysis we will frequently use a simple transformation by change of variables that can significantly simplify many technical derivations. To do so, we fix one point $r$ on the line $L$, and then introduce the following simple transformation by change of variables:

$$
\hat{x}=x-r .
$$

In terms of the $\hat{x}$-variable, the propagating field $E_{p}(x)$ in (19) takes the form

$$
E_{p}(x)=E_{p}(\hat{x}+r)=: \hat{E}_{p}(\hat{x})=s e^{i q \cdot(\hat{x}+r)}+\sum_{n \in \Xi_{0}} A^{n} e^{i q^{n} \cdot(\hat{x}+r)} .
$$

By setting $\hat{s}=s e^{i q \cdot r}$ and $\hat{A}^{n}=A^{n} e^{i q^{n} \cdot r}$, we can write $\hat{E}_{p}(\hat{x})$ into

$$
\hat{E}_{p}(\hat{x})=\hat{s} e^{i q \cdot \hat{x}}+\sum_{n \in \Xi_{0}} \hat{A}^{n} e^{i q^{n} \cdot \hat{x}} .
$$

A significant advantage of using the $\hat{x}$-variable, instead of the original $x$-variable, can be seen from the following lemma, where we write $\hat{s}$ for $\hat{A}^{0}$ and $q$ for $q^{0}$.

Lemma 3.8. Let $\Pi$ be a plane passing through the line $L$, the intersection line between $\Pi_{0}$ and $\Pi_{1}$ (see the beginning of Section 3.3 ). Then $\Pi \in \mathcal{P}_{0}$ if and only if $R_{\Pi}^{\prime} \mathcal{Q} \subset \mathcal{Q}$, and the relation $R_{\Pi}^{\prime} \hat{A}^{l}+\hat{A}^{m}=0$ holds whenever $R_{\Pi}^{\prime} q^{l}-q^{m}=0$ for $q^{l}, q^{m} \in \mathcal{Q}$.

Proof. We first note that $R_{\Pi} r=r$ since $\Pi$ passes through the line $L$. Then it follows that

$R_{\Pi} x=R_{\Pi}(\hat{x}+r)=R_{\Pi}^{\prime}(\hat{x}+r)+R_{\Pi} 0=R_{\Pi}^{\prime} \hat{x}+R_{\Pi}^{\prime} r+R_{\Pi} 0=R_{\Pi}^{\prime} \hat{x}+R_{\Pi} r=R_{\Pi}^{\prime} \hat{x}+r$.

Consequently,

$$
E_{p}\left(R_{\Pi} x\right)=\hat{E}_{p}\left(R_{\Pi}^{\prime} \hat{x}\right)
$$

As a result, the Reflection Principle (Lemma 2.1) can be written in terms of $\hat{E}_{p}(\hat{x})$ as follows. $\Pi \in \mathcal{P}_{0}$ if and only if the following equation holds:

$$
\hat{E}_{p}(\hat{x})+R_{\Pi}^{\prime}\left(\hat{E}_{p}\left(R_{\Pi}^{\prime}(\hat{x})\right)\right)=0 \quad \forall \hat{x} \in \mathbf{R}^{3} .
$$

Now, by substituting the expression (27) into the equation (28) and making use of Lemma 2.3, we obtain the desired results by comparing the coefficients of the Fourier modes.

3.5. Second class of unidentifiable gratings. We now start to consider the case when $L \|\left\{x_{3}=0\right\}$. By Lemma 3.7. we know $\left|\mathcal{P}_{0}\right| \in\{2,3\}$. We will study the case $\left|\mathcal{P}_{0}\right|=2$ in this subsection and the case $\left|\mathcal{P}_{0}\right|=3$ in the next subsection. These two cases will lead to two new classes of unidentifiable grating structures corresponding to the incident field $E^{i}(x)=s e^{i q \cdot x}$.

We first derive a few properties corresponding to the case $\left|\mathcal{P}_{0}\right|=2$.

Lemma 3.9. If $L \|\left\{x_{3}=0\right\}$ and $\left|\mathcal{P}_{0}\right|=2$, then it holds that

(1) $\mathcal{Q}=G\{q\}$ and $|G\{q\}|=2$. 
(2) $L \|\left(s \times e_{3}\right)$.

(3) $\mathcal{P}_{0}$ consists of exactly two planes, namely $\Pi_{0}$ and $\Pi_{1}$, where one is perpendicular to $s$ and the other is perpendicular to $s \times\left(s \times e_{3}\right)$. For ease of subsequent exposition, we shall assume (possibly after relabeling) that $\Pi_{0} \perp s$ and $\Pi_{1} \perp s \times\left(s \times e_{3}\right)$.

(4) Let $q^{1}=R_{\Pi_{1}}^{\prime} q=T_{\pi} q$. Then the propagating field $E_{p}(x)$ in (19) or $\hat{E}_{p}(\hat{x})$ in (27) can be written as

$$
E_{p}(x)=s\left(e^{i q \cdot x}-e^{i q^{1} \cdot x+i\left(q-q^{1}\right) \cdot r}\right) \quad \text { or } \quad \hat{E}_{p}(\hat{x})=\hat{s}\left(e^{i q \cdot \hat{x}}-e^{i q^{1} \cdot \hat{x}}\right) .
$$

Proof. It follows from Lemma 3.7 that $\mathcal{Q}=G\{q\}$. So we have $|G\{q\}|=2$ or $|G\{q\}|=4$ by Lemma 3.6(3). But one concludes from Lemma 3.6(4) that it is only possible to have $|G\{q\}|=2$, leading to (1).

By (1) and Lemma 3.5 we know that $\mathcal{P}_{0}=\left\{\Pi_{0}, \Pi_{1}\right\}$ and $\Pi_{0} \perp \Pi_{1}$. We know from Lemma 3.6 (3) that there exists a plane in $\mathcal{P}_{0}$, which we may assume to be $\Pi_{0}$ (possibly after relabeling the subscripts), such that $R_{\Pi_{0}}^{\prime} q=q$. Let $q^{1}=R_{\Pi_{1}}^{\prime} q$; we have by Lemma 3.6(3) that $\left\{q, q^{1}\right\}=G\{q\}=\mathcal{Q}$. Using this fact and the transformation (26), we can write $E_{p}(x)=\hat{E}_{p}(\hat{x})$ in (27) as

$$
\hat{E}_{p}(\hat{x})=\hat{s} e^{i q \cdot \hat{x}}+\hat{A}^{1} e^{i q^{1} \cdot \hat{x}} .
$$

Then we derive with the help of Lemma 3.8 and the relations $R_{\Pi_{0}}^{\prime} q=q$ and $q^{1}=R_{\Pi_{1}}^{\prime} q=T_{\pi} q$ that

$$
\hat{s}=-R_{\Pi_{0}}^{\prime} \hat{s}, \quad \hat{A}^{1}=-R_{\Pi_{1}}^{\prime} \hat{s} .
$$

The first relation in (30) implies $\hat{s} \perp \Pi_{0}$. Noting that $L$ lies on the plane $\Pi_{0}$, we have $\hat{s} \perp L$. But we know $L \perp e_{3}$ from the assumption, so $L \|\left(\hat{s} \times e_{3}\right)$. Observing that vector $s$ differs from $\hat{s}$ only by a scalar, we immediately obtain that $L \|\left(s \times e_{3}\right)$; hence we have proved (2) and (3). Finally, directly using the relations $\hat{s} \perp \Pi_{0}$ and $\Pi_{0} \perp \Pi_{1}$ we know that $\hat{s} \| \Pi_{1}$, which implies $\hat{A}^{1}=-R_{\Pi_{1}}^{\prime} \hat{s}=-\hat{s}$ and hence proves (4).

The next lemma indicates that all the perfect planes of the propagating field $E_{p}$ are determined by the incident field $E^{i}=s e^{i q \cdot x}$.

Lemma 3.10. If the line $L$ is parallel to the plane $\left\{x_{3}=0\right\}$ and $\left|\mathcal{P}_{0}\right|=2$, then

(1) All the perfect planes of $E_{p}$, i.e. the set $\mathcal{P}$, are determined by the incident field $E^{i}=s e^{i q \cdot x}$. More specifically, $\mathcal{P}$ consists of only two sets of planes: the first set contains only all the planes that are parallel to $\Pi_{0}$, while the second set contains only all the planes that are parallel to $\Pi_{1}$ and have the distance $\frac{\pi}{\left\|P_{\Gamma} q\right\|}$ between each two neighboring planes.

(2) Each face of the grating structure $S$ lies on a plane in $\mathcal{P}$.

Proof. We first show that all the planes in $\mathcal{P}$ are parallel to either $\Pi_{0}$ or $\Pi_{1}$. To see this, we consider a plane $\Pi \in \mathcal{P}$. By Proposition 3 we have $R_{\Pi}^{\prime} \mathcal{Q}=\mathcal{Q}$, i.e. $R_{\Pi}^{\prime}\left\{q, q^{1}\right\}=\left\{q, q^{1}\right\}$. Using Lemma 3.8, we know that $R_{\Pi}^{\prime} q=q$ implies $\Pi \| \Pi_{0}$ and $R_{\Pi}^{\prime} q=q^{1}$ implies $\Pi \| \Pi_{1}$.

Next, it is easy to see by the definition of a perfect plane and the expression (29) that all the planes parallel to $\Pi_{0}$ belong to $\mathcal{P}$. So it suffices to consider only the planes that are parallel to $\Pi_{1}$. Let $\Pi$ be such a plane in $\mathcal{P}$. By applying the 
relation (8) (Reflection Principle) to the plane $\Pi_{1}$ and $\Pi$, we obtain the following equations:

$$
E_{p}(x)+R_{\Pi_{1}}^{\prime}\left(E_{p}\left(R_{\Pi_{1}}(x)\right)\right)=0, \quad E_{p}(x)+R_{\Pi}^{\prime}\left(E_{p}\left(R_{\Pi}(x)\right)\right)=0 .
$$

Subtracting the second equation from the first one yields

$$
R_{\Pi_{1}}^{\prime}\left(E_{p}\left(R_{\Pi_{1}}(x)\right)\right)-R_{\Pi}^{\prime}\left(E_{p}\left(R_{\Pi}(x)\right)\right)=0 .
$$

Noting that $R_{\Pi_{1}}^{\prime}=R_{\Pi}^{\prime}$, the above relation reduces to

$$
E_{p}\left(R_{\Pi_{1}}(x)\right)-E_{p}\left(R_{\Pi}(x)\right)=0 .
$$

Hence if we substitute the expression (29) into this equation and make use of the equality $R_{\Pi} x=R_{\Pi}^{\prime} x+R_{\Pi} 0=R_{\Pi_{1}}^{\prime} x+R_{\Pi} 0$ and the symmetry of the reflection transformation $R_{\Pi_{1}}^{\prime}$, we further deduce that

$$
\begin{aligned}
& e^{i\left(R_{\Pi_{1}}^{\prime} q \cdot x+q \cdot R_{\Pi_{1}} 0\right)}-e^{i\left(R_{\Pi_{1}}^{\prime} q^{1} \cdot x+q^{1} \cdot R_{\Pi_{1}} 0+\left(q-q^{1}\right) \cdot r\right)} \\
= & e^{i\left(R_{\Pi}^{\prime} q \cdot x+q \cdot R_{\Pi} 0\right)}-e^{i\left(R_{\Pi}^{\prime} q^{1} \cdot x+q^{1} \cdot R_{\Pi} 0+\left(q-q^{1}\right) \cdot r\right)},
\end{aligned}
$$

which implies by using Lemma 2.3 that

$$
e^{i q \cdot\left(R_{\Pi} 0-R_{\Pi_{1}} 0\right)}=1, \quad e^{i q^{1} \cdot\left(R_{\Pi} 0-R_{\Pi_{1}} 0\right)}=1 .
$$

Now, we claim that $\left|q \cdot\left(R_{\Pi} 0-R_{\Pi_{1}} 0\right)\right|=\left|q^{1} \cdot\left(R_{\Pi} 0-R_{\Pi_{1}} 0\right)\right|=2 \mathrm{~d}\left(\Pi_{1}, \Pi\right)\left\|P_{\Gamma} q\right\|$, where $\mathrm{d}\left(\Pi_{1}, \Pi\right)$ is the distance between $\Pi_{1}$ and $\Pi$. Indeed, by using the relation $\nu \cdot \nu_{\Pi_{1}}=0$ and $R_{\Pi} 0-R_{\Pi_{1}} 0= \pm 2 \mathrm{~d}\left(\Pi_{1}, \Pi\right) \nu_{\Pi_{1}}$, we get $\left|q \cdot\left(R_{\Pi} 0-R_{\Pi_{1}} 0\right)\right|=$ $2 \mathrm{~d}\left(\Pi_{1}, \Pi\right)\left|P_{\Gamma} q \cdot \nu_{\Pi_{1}}\right|$ and $\left|q^{1} \cdot\left(R_{\Pi_{1}} 0-R_{\Pi_{1}} 0\right)\right|=2 \mathrm{~d}\left(\Pi_{1}, \Pi\right)\left|P_{\Gamma} q^{1} \cdot \nu_{\Pi_{1}}\right|$. But we know from Lemma 3.9 that $P_{\Gamma} q$ and $\nu_{\Pi_{1}}$ are parallel to each other since they are both perpendicular to the vector $s$ and $\nu$. Thus $\left|P_{\Gamma} q \cdot \nu_{\Pi_{1}}\right|=\left\|P_{\Gamma} q\right\|$, and our claim follows readily from the observation that $P_{\Gamma} q^{1}=T_{\pi} P_{\Gamma} q=-P_{\Gamma} q$. By the claim we know that the two equalities in (31) hold if and only if the distance between $\Pi$ and $\Pi_{1}$ is some multiple of $\frac{\pi}{\left\|P_{\Gamma} q\right\|}$. Therefore, we have shown that if $\Pi \| \Pi_{1}$ is a plane in $\mathcal{P}$, then $\mathrm{d}\left(\Pi_{0}, \Pi\right)$ equals some multiple of $\frac{\pi}{\left\|P_{\Gamma} q\right\|}$. On the other hand, if $\Pi$ is a plane such that $\Pi \| \Pi_{1}$ and $\mathrm{d}\left(\Pi_{0}, \Pi\right)$ equals some multiple of $\frac{\pi}{\left\|P_{\Gamma} q\right\|}$, then one can reverse the above deduction to show that equation (8) holds for all $x \in \mathbf{R}^{3}$. Then by the Reflection Principle, we conclude that $\Pi \in \mathcal{P}$. This completes the proof of the first part of the lemma.

Finally, we show the second part of the lemma. Let $\tilde{F}$ be an open face of the grating $S$ and let $F$ be the plane such that $\tilde{F} \subset F$. It suffices to prove that $F \subset$ $\mathcal{P}$. For this purpose, consider the four sequences of open faces $\left\{\tilde{F}+m \Lambda_{1} e_{1}\right\}_{m<0}$, $\left\{\tilde{F}+m \Lambda_{1} e_{1}\right\}_{m>0},\left\{\tilde{F}+m \Lambda_{2} e_{2}\right\}_{m<0}$ and $\left\{\tilde{F}+m \Lambda_{2} e_{2}\right\}_{m>0}$, all of which lie on $S$ due to the periodicity of the grating $S$. By the definition of a $\Pi_{0}$-reflecting periodic part, we see that for sufficiently large $m_{0}>0$, one of the sequences $\left\{\tilde{F}+m \Lambda_{1} e_{1}\right\}_{m<-m_{0}}$, $\left\{\tilde{F}+m \Lambda_{1} e_{1}\right\}_{m>m_{0}},\left\{\tilde{F}+m \Lambda_{2} e_{2}\right\}_{m<-m_{0}}$ and $\left\{\tilde{F}+m \Lambda_{2} e_{2}\right\}_{m>m_{0}}$ belongs to the $\Pi_{0^{-}}$ reflecting periodic part of $S$. Let $\left\{\tilde{F}+m \Lambda_{1} e_{1}\right\}_{m<-m_{0}}$ be such a sequence. Then Proposition 4 implies that all the open faces $\left\{\tilde{F}+m \Lambda_{1} e_{1}\right\}$ for $m<-m_{0}$ lie on planes in $\mathcal{P}$. Choose $n_{0}>m_{0}$; then $\tilde{F}$ is the reflection of $\tilde{F}-2 n_{0} \Lambda_{1} e_{1}$ with respect to $\tilde{F}-n_{0} \Lambda_{1} e_{1}$. By the Reflection Principal (Lemma 2.1) again, we know $\tilde{F}$ is a perfect plane of $E_{p}$, and so is the plane $F$, namely $F \in \mathcal{P}$. This completes the proof of Lemma 3.10. 
Lemma 3.10 enables us to find a new class of unidentifiable grating profiles. To describe the class explicitly, we first clarify some notation:

$r$ : a position vector, viewed as a point in $\mathbf{R}^{3}$;

$\Gamma$ : a plane which passes through the origin with normal $s \times e_{3}$;

$\Pi_{0}$ : a plane which passes through $r$ with normal $s$;

$\Pi_{1}$ : a plane which passes through $r$ with normal $\left(s \times e_{3}\right) \times s$.

Then by Lemma 3.10 all the perfect planes of $E_{p}$ can be described by

$$
\begin{gathered}
\mathcal{P}=\left\{\text { plane } \Pi ; \Pi \| \Pi_{0}\right\} \cup\left\{\text { plane } \Pi ; \Pi \| \Pi_{1}, \operatorname{dist}\left(\Pi, \Pi_{1}\right)=\frac{m \pi}{\left\|P_{\Gamma} q\right\|}\right. \\
\text { for some } m \in \mathbf{N}\} .
\end{gathered}
$$

This suggests a new class of unidentifiable gratings corresponding to the incident field $E^{i}=s e^{i q \cdot x}$ :

$$
\begin{array}{r}
\mathcal{S}_{1}(s, q, \Lambda, r)=\{\text { gratings with profile } S \text {, which are } \Lambda \text {-periodic polyhedral } \\
\text { structures such that faces of } S \text { lie on planes in } \mathcal{P}\} .
\end{array}
$$

One can see that each class $\mathcal{S}_{1}(s, q, \Lambda, r)$ corresponds to a unique electric field $E_{p}$, which solves the direct scattering problem (11)-(14) for any gratings in $\mathcal{S}_{1}(s, q, \Lambda, r)$. So any two grating in $\mathcal{S}_{1}(s, q, \Lambda, r)$ cannot be identified by the incident field $E^{i}(x)=$ $s e^{i q \cdot x}$. This proves

Lemma 3.11. If the line $L$ is parallel to $\left\{x_{3}=0\right\}$ and $\left|\mathcal{P}_{0}\right|=2$, then the grating profile $S$ belongs to $\mathcal{S}_{1}(s, q, \Lambda, r)$ for some point $r \in \mathbf{R}^{3}$. Furthermore, all gratings in $\mathcal{S}_{1}(s, q, \Lambda, r)$ generate the same total field.

Next we give a concrete example which has a non-empty class $\mathcal{S}_{1}(s, q, \Lambda, r)$.

Example 1. Let $\Lambda=\left(\Lambda_{1}, \Lambda_{2}\right)=\left(\frac{2 \pi}{\beta}, 0\right), q=\left(\alpha_{1}, \alpha_{2},-\beta\right)=\left(-\beta, \alpha_{2},-\beta\right), s=$ $e_{1}-e_{3}=(1,0,-1)$ and $r$ be the origin. Then we can check by direct computations that $\left(s \times e_{3}\right) \| e_{2}$ and

$$
q^{1}=T_{\pi} q=\left(\beta, \alpha_{2}, \beta\right)=q^{(2,0)}=\left(\alpha^{(2,0)}+\alpha\right)+\left(0,0, \beta^{(2,0)}\right) .
$$

In this case $\mathcal{P}$ consists of two sets of planes: the first set contains planes that are perpendicular to the vector $s=e_{1}-e_{3}$, and the second set contains planes that are perpendicular to the vector $e_{1}+e_{3}$ and have the distance $\frac{\pi}{\left\|P_{\Gamma} q\right\|}=\frac{\sqrt{2} \pi}{\beta}$ between each two neighboring planes.

Next we try to find some $\Lambda$-periodic structures in $\mathcal{P}$. For each $l \in \mathbf{Z}$, we denote by $L_{2 l}$ and $L_{2 l+1}$ the line of the form $\left\{\left(\frac{2 l \pi}{\beta}, \lambda, 0\right) ; \lambda \in \mathbf{R}\right\}$ and $\left\{\left(\frac{(2 l+1) \pi}{\beta}, \lambda, \frac{\pi}{\beta}\right) ; \lambda \in \mathbf{R}\right\}$, respectively. For any $m \in \mathbf{Z}$, let $\Pi_{m}$ be the plane that passes through the lines $L_{m}$ and $L_{m+1}$, and let $F_{m}$ be the part on $\Pi_{m}$ which lies between the lines $L_{m}$ and $L_{m+1}$. Then it is clear that $\Pi_{m}$ belongs to $\mathcal{P}$ for all $m \in \mathbf{Z}$ and that $\bigcup_{m \in \mathbf{Z}} F_{m}$ forms a $\Lambda$-periodic structure in $\mathcal{S}_{1}(s, q, \Lambda, r)$. By appropriate translations, we can get infinitely many $\Lambda$-periodic structures in $\mathcal{S}_{1}(s, q, \Lambda, r)$.

3.6. Third class of unidentifiable gratings. Continuing the discussion in the previous subsection, we now consider the case when $L \|\left\{x_{3}=0\right\}$ and $\left|\mathcal{P}_{0}\right|=3$. This case will lead to the third class of unidentifiable gratings corresponding to the given incident field $E^{i}$. 
Lemma 3.12. If the line $L$ is parallel to the plane $\left\{x_{3}=0\right\}$ and $\left|\mathcal{P}_{0}\right|=3$, then we have

(1) $\mathcal{Q}=G\{q\}$ and $|G\{q\}|=3$.

(2) $L \|\left(s \times e_{3}\right)$.

(3) $\mathcal{P}_{0}$ consists of only three planes: one is perpendicular to $s$, which we denote by $\Pi_{0}$, and the other two are generated by rotating the plane $\Pi_{0}$ about the axis $L$ by angles $\frac{\pi}{3}$ and $\frac{2 \pi}{3}$ respectively, which are labeled as $\Pi_{1}$ and $\Pi_{2}$.

(4) The propagating field $E_{p}$ in (19) or $\hat{E}_{p}$ in (27) can be written as

$$
\begin{aligned}
E_{p}(x) & =s e^{i q \cdot x}-\left(T_{\frac{2 \pi}{3}} s\right) e^{i q^{1} \cdot x+\left(q-q^{1}\right) \cdot r}+\left(T_{\frac{4 \pi}{3}} s\right) e^{i q^{2} \cdot x+\left(q-q^{2}\right) \cdot r}, \\
\hat{E}_{p}(\hat{x}) & =\hat{s} e^{i q \cdot \hat{x}}-\left(T_{\frac{2 \pi}{3}} \hat{s}\right) e^{i q^{1} \cdot \hat{x}}+\left(T_{\frac{4 \pi}{3}} \hat{s}\right) e^{i q^{2} \cdot \hat{x}}, \\
\text { where } q^{j} & =R_{\Pi_{j}}^{\prime} q=T_{\frac{2 j \pi}{3}} q \text { for } j=1,2 .
\end{aligned}
$$

Proof. The proof of (1) is the same as that of Lemma 3.9 (1). To prove the rest, we may write $\mathcal{P}_{0}=\left\{\Pi_{0}, \Pi_{1}, \Pi_{2}\right\}$ due to the fact that $\left|\mathcal{P}_{0}\right|=3$. Also, we know that each two neighboring planes in $\mathcal{P}_{0}$ form an angle of $\pi / 3$. By Lemma 3.6(3), there exists a plane, say $\Pi$, in $\mathcal{P}_{0}$ such that $R_{\Pi}^{\prime} q=q$. Without loss of generality, we may assume that this plane is $\Pi_{0}$. For $j=1,2$, let

$$
q^{j}=R_{\Pi_{j}}^{\prime} q=R_{\Pi_{j}}^{\prime}\left(R_{\Pi_{0}}^{\prime} q\right)=\left(R_{\Pi_{j}}^{\prime} \circ R_{\Pi_{0}}^{\prime}\right) q=T_{\frac{2 j \pi}{3}} q .
$$

Then it follows from part (1) that $\left\{q, q^{1}, q^{2}\right\}=G\{q\}=\mathcal{Q}$, and we can write $\hat{E}_{p}$ in (27) as

$$
\hat{E}_{p}(\hat{x})=\hat{s} e^{i q \cdot \hat{x}}+\hat{A}^{1} e^{i q^{1} \cdot \hat{x}}+\hat{A}^{2} e^{i q^{2} \cdot \hat{x}} .
$$

Now using Lemma 3.8 and the relation $q^{j}=R_{\Pi_{j}}^{\prime} q$ we deduce that

$$
\hat{s}=-R_{\Pi_{0}}^{\prime} \hat{s}, \quad \hat{A}^{1}=-R_{\Pi_{1}}^{\prime} \hat{s}, \quad \hat{A}^{2}=-R_{\Pi_{2}}^{\prime} \hat{s} .
$$

The first equation in (35) implies $\hat{s} \perp \Pi_{0}$ and further yields

$$
\begin{aligned}
& \hat{A}^{1}=-R_{\Pi_{1}}^{\prime} \hat{s}=\left(R_{\Pi_{1}}^{\prime} \circ R_{\Pi_{0}}^{\prime}\right) \hat{s}=T_{2 \pi / 3} \hat{s}, \\
& \hat{A}^{2}=-R_{\Pi_{2}}^{\prime} \hat{s}=\left(R_{\Pi_{2}}^{\prime} \circ R_{\Pi_{0}}^{\prime}\right) \hat{s}=T_{4 \pi / 3} \hat{s} .
\end{aligned}
$$

Finally a similar argument to that of Lemma 3.9 (2) leads to the relation $L \|\left(s \times e_{3}\right)$. This proves Lemma 3.12 .

Similar to the proof of Lemma 3.10, we can derive

Lemma 3.13. If the line $L$ is parallel to the plane $\left\{x_{3}=0\right\}$ and $\left|\mathcal{P}_{0}\right|=3$, then

(1) all the perfect planes of $E_{p}$, namely the set $\mathcal{P}$, are determined by the incident field $E^{i}=s e^{i q \cdot x}$. More specifically, $\mathcal{P}$ consists of only three sets of parallel planes, where every two neighboring planes in each set have equal distance $\frac{2 \pi}{\sqrt{3}\left\|P_{\Gamma} q\right\|}$ : the first set contains only the planes that are parallel to $\Pi_{0}$, the second set contains only the planes that are parallel to $\Pi_{1}$, while the third set contains only the planes that are parallel to $\Pi_{2}$.

(2) Each face of the grating structure $S$ lies on a plane in $\mathcal{P}$.

Lemma 3.13 leads us to a new class of unidentifiable grating profiles corresponding to the incident field $E^{i}=s e^{i q \cdot x}$. To describe this class explicitly, we first clarify 
some notation:

$r$ : a position vector, viewed as a point in $\mathbf{R}^{3}$;

$\Gamma$ : a plane which passes through the origin with normal $s \times e_{3}$;

$\Pi_{0}$ : a plane which passes through $r$ with normal $s$;

$\Pi_{1}, \Pi_{2}$ : planes which pass through $r$ and form an angle of $\pi / 3$ and $2 \pi / 3$ with $\Pi_{0}$, respectively.

Then by Lemma 3.13, we can describe all the perfect planes of $E_{p}$ in (19) by

$$
\begin{gathered}
\mathcal{P}=\left\{\text { plane } \Pi ; \exists j \in\{0,1,2\} \text { such that } \Pi \| \Pi_{j}, \operatorname{dist}\left(\Pi, \Pi_{j}\right)=\frac{2 m \pi}{\sqrt{3}\left\|P_{\Gamma} q\right\|}\right. \\
\text { for some } m \in \mathbf{N}\},
\end{gathered}
$$

which suggests a new class of unidentifiable gratings corresponding to the incident field $E^{i}$ :

$$
\begin{array}{r}
\mathcal{S}_{2}(s, q, \Lambda, r)=\{\text { gratings with profile } S, \text { which are } \Lambda \text {-periodic polyhedral } \\
\text { structures such that faces of } S \text { lie on planes in } \mathcal{P}\} .
\end{array}
$$

One can see that each class $\mathcal{S}_{2}(s, q, \Lambda, r)$ corresponds to a unique propagating field $E_{p}$, which solves the direct scattering problem (11)-(41) for any grating in $\mathcal{S}_{2}(s, q, \Lambda, r)$. Thus any two gratings in $\mathcal{S}_{2}(s, q, \Lambda, r)$ cannot be identified by the incident field $E^{i}(x)=s e^{i q \cdot x}$. This leads to the following lemma.

Lemma 3.14. If the line $L$ is parallel to the plane $\left\{x_{3}=0\right\}$ and $\left|\mathcal{P}_{0}\right|=3$, then the grating $S$ belongs to $\mathcal{S}_{2}(s, q, \Lambda, r)$ for some point $r \in \mathbf{R}^{3}$. Furthermore, all the gratings in $\mathcal{S}_{2}(s, q, \Lambda, r)$ generate the same total field.

Next we give a concrete example which has a non-empty class $\mathcal{S}_{2}(s, q, \Lambda, r)$.

Example 2. Let $\Lambda=\left(\Lambda_{1}, \Lambda_{2}\right)=\left(\frac{4 \pi}{\sqrt{3}}, 0\right), q=\left(\alpha_{1}, \alpha_{2},-\beta\right)=(0,0,-1), s=e_{1}$ and $r$ be the origin. Then we can check by direct computations that $\left(s \times e_{3}\right) \| e_{2}$ and

$$
\begin{aligned}
& q^{1}=\left(\frac{\sqrt{3}}{2}, 0, \frac{1}{2}\right)=q^{(1,0)}=\left(\alpha^{(1,0)}+\alpha\right)+\left(0,0, \beta^{(1,0)}\right) \\
& q^{2}=\left(-\frac{\sqrt{3}}{2}, 0, \frac{1}{2}\right)=q^{(-1,0)}=\left(\alpha^{(-1,0)}+\alpha\right)+\left(0,0, \beta^{(-1,0)}\right) .
\end{aligned}
$$

Moreover, $\mathcal{P}$ consists of three sets of parallel planes, respectively with $e_{1},-e_{1}+$ $\sqrt{3} e_{3}$ and $-e_{1}+\sqrt{3} e_{3}$ as the normal directions, and the distance between any two neighboring parallel planes in each set is $\frac{2 \pi}{\sqrt{3}\left\|P_{\Gamma} q\right\|}=\frac{2 \pi}{\sqrt{3}}$.

Next we try to find some $\Lambda$-periodic structures in $\mathcal{P}$. For each $l \in \mathbf{Z}$, we denote by $L_{2 l}$ and $L_{2 l+1}$ the line of the form $\left\{\left(\frac{4 l \pi}{\sqrt{3}}, \lambda, 0\right) ; \lambda \in \mathbf{R}\right\}$ and $\left\{\left(\frac{2(2 l+1) \pi}{\sqrt{3}}, \lambda, \frac{2 \pi}{3}\right) ; \lambda \in\right.$ $\mathbf{R}$ \}, respectively. For any $m \in \mathbf{Z}$, let $\Pi_{m}$ be the plane that passes through the lines $L_{m}$ and $L_{m+1}$, and let $F_{m}$ be the part of $\Pi_{m}$ which lies between the lines $L_{m}$ and $L_{m+1}$. Then it is clear that $\Pi_{m}$ belongs to $\mathcal{P}$ for all $m \in \mathbf{Z}$ and that $\bigcup_{m \in \mathbf{Z}} F_{m}$ forms a $\Lambda$-periodic structure in $\mathcal{S}_{2}(s, q, \Lambda, r)$. By appropriate translations, we can obtain infinitely many $\Lambda$-periodic structures in $\mathcal{S}_{2}(s, q, \Lambda, r)$.

3.7. Excluding the case with $L \nVdash\left\{x_{3}=0\right\}$. We have discussed in Subsections 3.5 3.6 the case that the intersection line $L$ between two perfect planes $\Pi_{0}$ and $\Pi_{1}$ is parallel to the plane $\left\{x_{3}=0\right\}$, which has led to two non-trivial classes of unidentifiable grating structures. In this subsection we study the case when the 
line $L$ is not parallel to the plane $\left\{x_{3}=0\right\}$. As we will see, this case cannot happen. Recall that the $x_{3}$ component of $\nu$ is positive when $L \nVdash\left\{x_{3}=0\right\}$. We start with the following result that is the foundation of the analysis in this subsection.

Lemma 3.15. If $L \nVdash\left\{x_{3}=0\right\}$, then there exists a plane $\Pi^{*} \in \mathcal{P}$ such that $\Pi^{*} \nVdash L$.

Proof. Assume the lemma does not hold. Then all the perfect planes of $E_{p}$ are parallel to the line $L$. But by Proposition 4 each face of a $\Pi_{0}$-reflecting periodic part $S_{l}$ of grating $S$ lies on some perfect plane of $E_{p}$. Hence all the faces of $S_{l}$ are parallel to the line $L$, and so are all the faces of $S$ due to the periodic structure of $S$. Since $L \nVdash\left\{x_{3}=0\right\}$, the grating profile $S$ cannot be bounded in the $x_{3}$ direction, which is a contradiction.

Next, we show some useful relations for the subsequent analysis.

Lemma 3.16. If line $L$ is not parallel to the plane $\left\{x_{3}=0\right\}$, then it holds that

$$
\begin{aligned}
& q+\sum_{n \in \Xi_{0}} q^{n}=0, \\
& \tau+\sum_{n \in \Xi_{0}} \tau_{n}=0, \\
& \tau_{n}>0 \quad \forall q^{n} \in \mathcal{Q} \backslash G\{q\} .
\end{aligned}
$$

Proof. We know from Lemma 3.15 that there exists a plane $\Pi^{*} \in \mathcal{P}$ such that $\Pi^{*} \nVdash L$. To see (36), we set $Q=q+\sum_{n \in \Xi_{0}} q^{n}$. Recall that for any $\Pi \in \mathcal{P}$, $R_{\Pi}^{\prime} \mathcal{Q}=\mathcal{Q}$, so $R_{\Pi}^{\prime} Q=Q$ as $R_{\Pi}^{\prime}$ is bijective. Therefore, $Q \| \Pi$ for all $\Pi \in \mathcal{P}$. Especially, we have $Q\left\|\Pi_{0}, Q\right\| \Pi_{1}$, and $Q \| \Pi^{*}$. Hence $Q=0$ by noting that $L\left\|\Pi_{0}, L\right\| \Pi_{1}$, but $L \nmid \Pi^{*}$. It follows immediately that

$$
Q \cdot \nu=\tau+\sum_{n \in \Xi_{0}} \tau_{n}=0 .
$$

To see (38), consider the orbit $G\left\{q^{n}\right\}$ of $q^{n} \in \mathcal{Q} \backslash G\{q\}$ under the action of $G$; we have $G\left\{q^{n}\right\}=\tau_{n} \nu+G\left\{P_{\Gamma} q^{n}\right\}$. Referring to Lemma 3.6 (4), there exists at least one element of $G\left\{P_{\Gamma} q^{n}\right\}$ with non-positive $x_{3}$ component. There also exists at least one element in $G\left\{q^{n}\right\}$ if $\tau_{n} \leq 0$, which is in contradiction to Proposition 1. Therefore we have $\tau_{n}>0$.

Based on the following decomposition from (25),

$$
q=\tau \nu+P_{\Gamma} q,
$$

we are now going to separate the remaining arguments into two cases, $\tau=0$ and $\tau \neq 0$, and show that both cases cannot happen (Lemmas 3.17 3.18 and 3.19), thus concluding that the case when the line $L$ is not parallel to the plane $\left\{x_{3}=0\right\}$ cannot occur.

Lemma 3.17. If $L \nVdash\left\{x_{3}=0\right\}$, then $q$ has a non-zero projection on $\nu$, i.e., $\tau \neq 0$ (see (39)). 
Proof. We prove the lemma by contradiction. Assume $\tau=0$. Using (37) and (38), we know $\mathcal{Q} \backslash G\{q\}=\emptyset$, so we have $\mathcal{Q}=G\{q\}$. We now turn to exclude each possibility based on $|G\{q\}|$ :

(1) $|G\{q\}|>3$. By Lemma3.6(4), there are at least two elements in $G\{q\}$ whose $x_{3}$ components are not positive. This yields a contradiction to Proposition 1.

(2) $|G\{q\}|=3$. By Lemma 3.6(3), we should have $|G\{q\}|=\left|\mathcal{P}_{0}\right|=3$, which further yields that $G\{q\}=\left\{R_{\Pi}^{\prime} q: \Pi \in \mathcal{P}_{0}\right\}$. Let $\Pi^{*}$ be the plane mentioned in Lemma 3.15. Then it follows from Proposition 3 that $R_{\Pi^{*}}^{\prime} q \in \mathcal{Q}=G\{q\}$, and thus there exists a plane $\Pi \in \mathcal{P}_{0}$ such that $R_{\Pi^{*}}^{\prime} q=R_{\Pi}^{\prime} q$. Using Lemma 3.2. we know that $\Pi \| \Pi^{*}$, giving contradiction to the facts that $\Pi \| L$ but $\Pi^{*} \nVdash L$.

(3) $|G\{q\}|=2$. It can be shown in the same argument as that for the case of $|G\{q\}|=3$.

Using Lemma 3.17, we need only to consider the case with $\tau \neq 0$.

Lemma 3.18. If $L \nVdash\left\{x_{3}=0\right\}$, it cannot happen that $|G\{q\}| \geq 3$ and $\tau \neq 0$.

Proof. We first exclude the case with $|G\{q\}|>3$ and $\tau \neq 0$. Noting $\tau_{n}=\tau$ for any $q^{n} \in G\{q\}$ (Lemma 3.6(1)) and the equalities (37) and (38), we know that $\tau_{n} \neq 0$, so it is the $x_{3}$ component of the vector $\tau \nu$. Now consider the set $G\{q\}=\tau \nu+G\left\{P_{\Gamma} q\right\}$. By Lemma 3.6 (4), there exist at least two elements in $G\left\{P_{\Gamma} q\right\}$ whose $x_{3}$ components are non-positive. Hence there are at least two elements in $G\{q\}$ which have nonpositive $x_{3}$ components, yielding a contradiction to Proposition 1

We next exclude the case with $|G\{q\}|=3$ and $\tau \neq 0$. By equality (37) and the fact that $\tau \neq 0$, we know that $\mathcal{Q} \backslash G\{q\} \neq \emptyset$. So there exists some $q^{m} \in \mathcal{Q} \backslash G\{q\}$ such that $\tau_{m}>0$ by (38). As $|G\{q\}|=3$, we have by Lemma 3.6. (3) that $\left|\mathcal{P}_{0}\right|=3$ and $\left|G\left\{q^{m}\right\}\right| \geq 3$; in fact $\left|G\left\{q^{m}\right\}\right|=3$ or $=6$. Now, noting $\tau_{n}=\tau$ for all $q^{n} \in G\{q\}$, we have

$$
3 \tau+3 \tau_{m} \leq \tau+\sum_{q^{n} \in G\{q\}} \tau_{n}+\sum_{q^{n} \in G\left\{q^{m}\right\}} \tau_{n} \leq \tau+\sum_{q^{n} \in \mathcal{Q}} \tau_{n}=\tau+\sum_{n \in \Xi_{0}} \tau_{n}=0 .
$$

Define

$$
d_{0}=\min \left\{x_{3} ; x=\left(x_{1}, x_{2}, x_{3}\right) \in \Gamma \text { and }\|x\| \leq\left\|P_{\Gamma} q\right\|\right\}<0 .
$$

We can find $q^{n_{1}} \in G\{q\} \backslash\{q\}$ such that the $x_{3}$ component of $P_{\Gamma} q^{n_{1}}$ is less than or equal to $-\frac{1}{2} d_{0}$. Since $\left\|q^{m}\right\|=\|q\|=k$ and $\tau+\tau_{m} \leq 0$, we deduce $\left\|P_{\Gamma} q^{m}\right\| \geq\left\|P_{\Gamma} q\right\|$. We can find $q^{n_{2}} \in G\left\{q^{m}\right\}$ such that the $x_{3}$ component of $P_{\Gamma} q^{n_{2}}$ is less than or equal to $\frac{1}{2} d_{0}$. Then the $x_{3}$ component of

$$
q^{n_{1}}+q^{n_{2}}=\left(\tau+\tau_{m}\right) \nu+P_{\Gamma} q^{n_{1}}+P_{\Gamma} q^{n_{2}}
$$

is non-positive, which is in contradiction to Proposition 1

Lemma 3.19. If $L \nVdash\left\{x_{3}=0\right\}$, then it cannot happen that $|G\{q\}| \leq 2$ and $\tau \neq 0$.

Proof. First by Lemma3.6(3) we know that $G\{q\} \geq 2$, so it suffices to show that the case with $|G\{q\}|=2$ and $\tau \neq 0$ cannot occur. We will prove this by contradiction. By Lemma 3.15, there exists a perfect plane $\Pi^{*} \in \mathcal{P}$ such that $\Pi^{*} \nVdash L$. We shall derive contradictions for each possibility specified by the geometric relations among the planes $\Pi^{*}$ and $\Pi_{0}, \Pi_{1}$. 
Case 1. $\Pi^{*} \perp \Pi_{0}, \Pi^{*} \perp \Pi_{1}$. Since $|G\{q\}|=2$, we may write $G\{q\}=\left\{q, q^{*}\right\}$. Clearly there are only two reflections in $G$, and we know that $q^{*}=\tau \nu-P_{\Gamma} q$ if $q=$ $\tau \nu+P_{\Gamma} q$. As $\Pi^{*} \perp \Pi_{0}$ and $\Pi_{1}$, we derive $R_{\Pi^{*}}^{\prime} G\{q\}=\left\{-\tau \nu+P_{\Gamma} q,-\tau \nu-P_{\Gamma} q\right\} \subset \mathcal{Q}$. This shows that both $q^{*}$ and $-q^{*}$ belong to $\mathcal{Q}$. As a result one of them must have a non-positive $x_{3}$ component, which is in contradiction to Proposition 1 .

Case 2. $\Pi^{*} \not \perp \Pi_{0}$. Let $\tilde{L}, \tilde{\mathcal{P}_{0}}, \tilde{G}$ denote the line of intersection between $\Pi^{*}$ and $\Pi_{0}$, perfect planes in $\mathcal{P}$ which pass through the line $\tilde{L}$, and the group generated by the reflections $\left\{R_{\Pi}^{\prime}: \Pi \in \tilde{\mathcal{P}}_{0}\right\}$. Since $\Pi^{*} \not \perp \Pi_{0}$, we see that $\left|\tilde{\mathcal{P}}_{0}\right| \geq 3$, and hence $|\tilde{G}\{q\}| \geq 3$ by following the proof of Lemma 3.6 (3).

We first consider the case when $\tilde{L}$ is not parallel to the plane $\left\{x_{3}=0\right\}$. In this case we have $|\tilde{G}\{q\}| \geq 3$. Then one can deduce a contradiction by following the same arguments as those in Lemmas 3.17 and 3.18 .

We then consider the case when $\tilde{L} \|\left\{x_{3}=0\right\}$. This is the same situation as we have addressed in Lemma 3.7. Subsection 3.3 and the entire Subsection 3.6. We see that the current case can occur only when $|\tilde{G}\{q\}|=|\tilde{G}|=3$. Following the steps in Subsection 3.6. we can work out all the perfect planes in $\mathcal{P}$. As shown in the case considered in Subsection 3.6, the intersection lines of the planes in $\mathcal{P}$ are all parallel to $\tilde{L}$. In particular, we have $L \| \tilde{L}$; thus $L \|\left\{x_{3}=0\right\}$, which is in contradiction to the assumption of Lemma 3.19

Case $3 . \Pi^{*} \not \perp \Pi_{1}$. The same argument as that for Case 2 above leads to a contradiction.

This completes the proof of Lemma 3.18

One can readily conclude from Lemmas 3.173 .18 and 3.19 that the case considered in this subsection when the line $L$ is not parallel to the plane $\left\{x_{3}=0\right\}$ cannot occur.

3.8. Summary on all unidentifiable gratings. Summing up the results in Subsection 3.2 3.3 and 3.5]3.7] especially Lemmas 3.3, 3.11] 3.14] and Lemmas 3.17]3.19] we come to the following conclusion.

Theorem 3.1. Let $S$ be a polyhedral grating with bi-period $\Lambda, E^{i}(x)=s e^{i q \cdot x}$ be an incident electric field, and $E$ be a solution to the direct scattering problems (11)-(4). Then under condition (6) and Assumption 1, we have

$$
S \in \mathcal{S}_{0}(q, r) \quad \text { or } \quad S \in \mathcal{S}_{1}(s, q, \Lambda, r) \quad, \quad S \in \mathcal{S}_{2}(s, q, \Lambda, r) .
$$

\section{Unique DEtermination of a PERIOdic POLYhedRAL GRATING PROFILE}

In this section we apply the results developed in the previous section on the classification of unidentifiable periodic grating structures in correspondence to one incident field for the unique determination of a given periodic polyhedral grating profile by the scattered field.

Theorem 4.1. Let $E^{i}=s e^{i q \cdot x}$ be a given incident electric field, $S_{1}$ and $S_{2}$ be two periodic polyhedral gratings with bi-period $\Lambda$, and $E_{1}$ and $E_{2}$ be respectively the solutions to the forward scattering problem (11)-(44) associated with $S_{1}$ and $S_{2}$. If $\Gamma_{b}=\left\{x_{3}=b\right\}$ is a plane located above both $S_{1}$ and $S_{2}$, then under condition (6) the information

$$
e_{3} \times E_{1}=e_{3} \times E_{2} \quad \text { on } \Gamma_{b}
$$


implies one of the following three cases for some point $r \in \mathbf{R}^{3}$ :

(a) $S_{1}, S_{2} \in \mathcal{S}_{0}(q, r)$; (b) $S_{1}, S_{2} \in \mathcal{S}_{1}(s, q, \Lambda, r)$; (c) $S_{1}, S_{2} \in \mathcal{S}_{2}(s, q, \Lambda, r)$.

Proof. Assume that (40) is true for two different $S_{1}$ and $S_{2}$. We first show that $E_{1}=$ $E_{2}$ in the domain above the measurement plane $\left\{x_{3}=b\right\}$. Using the expansions

$$
\begin{aligned}
& E_{1}(x)=E^{i}(x)+\sum_{n \in \mathbf{Z}^{2}} A_{1}^{n} e^{i q^{n} \cdot x}, \\
& E_{2}(x)=E^{i}(x)+\sum_{n \in \mathbf{Z}^{2}} A_{2}^{n} e^{i q^{n} \cdot x},
\end{aligned}
$$

it suffices to show that $A_{1}^{n}=A_{2}^{n}$ for all $n \in \mathbf{Z}^{2}$. To see this, we have by (40) that

$$
\left(E_{1}-E_{2}\right) \times\left. e_{3}\right|_{x_{3}=b}=\sum_{n \in \mathbf{Z}^{2}}\left(A_{1}^{n}-A_{2}^{n}\right) \times e_{3} e^{i \beta^{n} \cdot b} e^{i \alpha^{n} \cdot x}=0 .
$$

Noting that $\left\{e^{i \alpha^{n} \cdot x}\right\}_{n \in \mathbf{Z}^{2}}$ is an orthogonal family in $L^{2}\left(\left(0, \Lambda_{1}\right) \times\left(0, \Lambda_{2}\right)\right)$ of variables $x_{1}$ and $x_{2}$, we derive from (41) that

$$
\left(A_{1}^{n}-A_{2}^{n}\right) \times e_{3}=0 \quad \forall n \in \mathbf{Z}^{2} .
$$

In addition, as $A_{1}^{n} \cdot q^{n}=0$ and $A_{2}^{n} \cdot q^{n}=0$, which can be deduced from the fact that both the fields $E_{1}$ and $E_{2}$ are divergence free, we have

$$
\left(A_{1}^{n}-A_{2}^{n}\right) \cdot q^{n}=0 \quad \forall n \in \mathbf{Z}^{2} .
$$

Now, if $A_{1}^{n}-A_{2}^{n} \neq 0$ for some $n \in \mathbf{Z}^{2}$, then we can conclude from (42) and (43) that

$$
q^{n} \cdot e_{3}=\beta^{n}=0,
$$

which is in contradiction to the assumption (6). Thus we have shown that $E_{1}=E_{2}$ in the domain above the plane $\left\{x_{3}=b\right\}$. Since both $E_{1}(x)$ and $E_{2}(x)$ are analytic functions, we see that $E_{1}(x)=E_{2}(x)$ in their common domain $S_{1}^{+} \cap S_{2}^{+}$, where $S_{i}^{+}$ is the domain in $\mathbf{R}^{3}$ above $S_{i}$ for $i=1,2$. As a result, faces of the grating profile $S_{2}$ which lie above $S_{1}$ are perfect planes of $E_{1}$, and faces of the grating profile $S_{1}$ which lie above $S_{2}$ are perfect planes of $E_{2}$. Now we may assume without loss of generality that the grating profile $S_{2}$ has some part above $S_{1}$. Then the Lipschitz condition we imposed on $S_{2}$ allows us to find a face (referring to an open part of a plane) on $S_{2}$ which is above $S_{1}$ and is not parallel to $e_{3}$. By the previous discussions, this face is a perfect plane of $E_{1}$, which is denoted by $\Pi$.

One may notice that the perfect plane $\Pi$ may not necessarily extend above the measurement plane $\left\{x_{3}=b\right\}$. In order to apply Theorem 3.1, we need to construct a perfect plane which extends above the plane $\Gamma_{b}$ and which is not parallel to $e_{3}$. This is done in the following.

First we choose a perfect point $x_{0} \in \Pi$. Let $\gamma(t)$ be the ray emitted from $x_{0}$ and directed along $e_{3}$; more precisely, $\gamma(t)=x_{0}+t e_{3}$ for $t \geq 0$. Let $d_{0}>0$ be a number less than half of the distance between the ray $\gamma(t)$ and the grating profile $S_{1}$. Consider the ray $R_{\Pi} \gamma(t)$. If it does not intersect $S_{1}$, then it directs upward or horizontally and lies entirely above the grating profile $S_{1}$. So does its projection on the plane $\Pi$, which is then our desired perfect plane. Otherwise, the ray $R_{\Pi} \gamma(t)$ intersects $S_{1}$ at some point; let

$$
t_{1}=\min \left\{t \geq 0 ; R_{\Pi} \gamma(t) \text { belongs to } S_{1}\right\} .
$$

Clearly $t_{1}>d_{0}$ and $R_{\Pi} \gamma\left(t_{1}\right)$ belongs to a (closed) face of $S_{1}$, which is not parallel to $R_{\Pi}^{\prime} e_{3}$. By the Reflection Principle (Lemma 2.1), $\gamma\left(t_{1}\right)$ is a perfect point of $E_{1}$ and has a perfect plane, say $\Pi_{1}$, which passes through $\gamma\left(t_{1}\right)$ but $\Pi_{1} \nVdash e_{3}$. We can do the same procedure for $\gamma\left(t_{1}\right)$ and $\Pi_{1}$ as we did for $\gamma\left(t_{0}\right)=\gamma(0)$ and $\Pi$ above. Then 
either $\Pi_{1}$ is our desired perfect plane or we can find another perfect point $\gamma\left(t_{2}\right)$ and perfect plane $\Pi_{2}$ of $E_{1}$ such that $\Pi_{2}$ passes through $\gamma\left(t_{2}\right)$ and $\Pi_{2} \nVdash e_{3}$. Clearly we see that $t_{2}-t_{1}>d_{0}$. Repeating this procedure, we can get a sequence of perfect points $\left\{\gamma\left(t_{n}\right)\right\}$ and perfect planes $\left\{\Pi_{n}\right\}$ of $E_{1}$ such that $\Pi_{n}$ passes through $\gamma\left(t_{n}\right)$ and $\Pi_{n} \nVdash e_{3}$, and $t_{n}-t_{n-1}>d_{0}$. So after a finite number of steps we can get a desired perfect plane of $E_{1}$, denoted as $\Pi^{*}$, that extends above $\Gamma_{b}$. Since $E_{1}$ and $E_{2}$ coincide in $S_{1}^{+} \cap S_{2}^{+}, \Pi^{*}$ is a perfect plane for both $E_{1}$ and $E_{2}$. Now Theorem 4.1 follows from Theorem 3.1

Theorem 4.1 leads immediately to the following two corollaries.

Corollary 4.1. Let $S$ be a polyhedral grating of bi-period $\Lambda$. Consider a plane $\Gamma_{b}=\left\{x_{3}=b\right\}$ located above $S$. Then the measurement of the total field $e_{3} \times E$ on $\Gamma_{b}$ corresponding to the incident field $E^{i}$ determines $S$ uniquely if the following condition holds:

There are two faces of $S$ which do not form an angle of $\pi / 3, \pi / 2$ or $2 \pi / 3$.

Corollary 4.2. Let $S$ be a polyhedral grating of bi-period $\Lambda$ which is not an entire plane in $R^{3}$, and let $E^{i, 1}=s_{1} e^{i q_{1} \cdot x}$ and $E^{i, 2}=s_{2} e^{i q_{2} \cdot x}$ be two incident fields. Let $\Gamma_{b}=\left\{x_{3}=b\right\}$ be a plane located above $S$. Then the measurement of the total fields $e_{3} \times E^{1}$ and $e_{3} \times E^{2}$ on $\Gamma_{b}$ which correspond respectively to the incident waves $E^{i, 1}$ and $E^{i, 2}$ determines $S$ uniquely if the following condition holds:

(a) The vectors $s_{1}$ and $s_{2}$ are not parallel, orthogonal or form an angle of $\pi / 3$ or $2 \pi / 3$.

Proof. For the incident waves $E^{i, 1}$ and $E^{i, 2}$, we define

$$
\begin{aligned}
& \mathcal{S}_{1}=\left(\bigcup_{r \in \mathbf{R}^{3}} \mathcal{S}_{1}\left(s_{1}, q_{1}, \Lambda, r\right)\right) \cup\left(\bigcup_{r \in \mathbf{R}^{3}} \mathcal{S}_{2}\left(s_{1}, q_{1}, \Lambda, r\right)\right), \\
& \mathcal{S}_{2}=\left(\bigcup_{r \in \mathbf{R}^{3}} \mathcal{S}_{1}\left(s_{2}, q_{2}, \Lambda, r\right)\right) \cup\left(\bigcup_{r \in \mathbf{R}^{3}} \mathcal{S}_{2}\left(s_{2}, q_{2}, \Lambda, r\right)\right) .
\end{aligned}
$$

Then by Theorem 4.1 a grating profile $S$ which is not parallel to $\left\{x_{3}=0\right\}$ cannot be identified by both $E^{i, 1}$ and $E^{i, 2}$ if and only if $S \in \mathcal{S}_{1} \cap \mathcal{S}_{2}$. We clearly see that if condition (a) holds, then $\mathcal{S}_{1} \cap \mathcal{S}_{2}=\emptyset$, which immediately leads to the corollary.

One can see from Corollaries 4.1 and 4.2 that a general polyhedral grating structure can be uniquely determined by one or two incident plane waves. Only gratings of very special structures may require more incident waves for their unique determination.

\section{REFERENCES}

1. T. Abboud and J. C. Nédélec, Electromagnetic waves in an inhomogeneous medium, J. Math. Anal. Appl., 164 (1992), 40-58. MR.1146575 (93a:78005)

2. G. Alessandrini and L. Rondi, Determining a sound-soft polyhedral scatterer by a single farfield measurement, Proc. Amer. Math. Soc., 6 (2005), 1685-1691. MR2120253 (2005i:35276)

3. H. Ammari, Théorèmes d'unicité pour un problème inverse dans une structure bipériodique, C. R. Acad. Sci. Paris, Série I, t. 320 (1995), 815-820. MR1326688 (96a:78007)

4. G. Bao, L. Cowsar and W. Masters, Mathematical Modeling in Optical Science, SIAM Philadelphia, 2001. MR:1831328 (2001m:78002)

5. G. Bao, Variational approximation of Maxwell's equations in biperiodic structrues, SIAM J. Appl. Math., 57 (1997), 364-381. MR1438758 (97m:65199)

6. G. Bao and D. Dobson, On the scattering by a biperiodic structure, Proc. Amer. Math. Soc., 128 (2000), 2715-2723. MR 1694448 (2000m:35051) 
7. G. Bao and A. Friedman, Inverse problems for scattering by periodic structures, Arch. Rat. Mech. Anal., 132 (1995), 49-72. MR.1360080 (96i:35131)

8. G. Bao and Z. Zhou, An inverse problem for scattering by a doubly periodic structure, Trans. Amer. Math. Soc., 350 (1998), 4089-4103. MR1487607(99b:78010)

9. J. Cheng and M. Yamamoto, Uniqueness in an inverse scattering problem within non-trapping polygonal obstacles with at most two incoming waves, Inverse Problems, 19 (2003), 1361-1384. MR:2036535 (2004k:35394)

10. D. Colton and R. Kress, Integral Equation Method in Scattering Theory, John Wiley \& Sons, Inc. 1983. MR.700400 (85d:35001)

11. D. Colton and R. Kress, Inverse Acoustic and Electromagnetic Scattering Theory, Second Edition, Springer-Verlag, Berlin, 1998. MR1635980 (99c:35181)

12. D. Dobson and A. Friedman, The time-harmonic Maxwell equations in a doubly periodic structure, J. Math. Anal. Appl., 166 (1992), 507-528. MR.1160941 (92m:78015)

13. Electromagnetic Theory of Gratings, Topics in Current Physics, Vol. 22, R. Petit, Editor, Springer-Verlag, Heidelberg, 1980. MR609533 (82a:78001)

14. J. Elschner, G. Schmidt and M. Yamamoto, An inverse problem in periodic diffractive optics: Global uniqueness with a single wavenumber, Inverse Problems, 19 (2003), 779-787. MR 1984889 (2005c:78018)

15. J. Elschner, G. Schmidt and M. Yamamoto, Global uniqueness in determining rectanglular periodic structures by scattering data with a single wave number, J. Inverse Ill-posed Probl, 11 (2003), 235-244. MR2001731 (2004i:78016)

16. J. Elschner, G.Schmidt, Inverse scattering for periodic structures: Stability of polygonal interfaces, Inverse Problems, 17 (2001), 1817-1829. MR.1872923(2004e:78025)

17. J. Elschner and M. Yamamoto, Uniqueness in determining polygonal sound-hard obstacles with a single incoming wave, Inverse Problems, 22 (2006), 355-364. MR2194199 (2006j:35240)

18. J. Elschner and M. Yamamoto, Uniqueness in determing polygonal periodic structures, Zeits. Anal. Anwendungen, 26 (2007), 165-177. MR2314159 (2008h:35367)

19. F. Hettlich and A. Kirsch, Schiffer's theorem in inverse scattering for periodic structures, Inverse Problems, 13 (1997), 351-361. MR 1445923 (98d:35034)

20. T.W. Hungerford, Algebra, Springer, New York, 1974. MR0354211 (50:6693)

21. A. Kirsch and R. Kress, Uniqueness in inverse obstacle scattering, Inverse Problems, 9 (1993), 285-299. MR1214288 (94e:35143)

22. H.Y.Liu, M. Yamamoto and J.Zou, Reflection principle for the Maxwell equations and its application to inverse electromagnetic scattering, Inverse Problem, 23 (2007), 2357-2366. MR2441007 (2009g:78020)

23. H. Y.Liu and J.Zou, Uniqueness in an inverse acoustic obstacle scattering problem for both sound-hard and sound-soft polyhedral scatterers, Inverse Problems, 22 (2006), 515-524. MR2216412 (2007b:35332)

Department of Mathematics, Michigan State University, East Lansing, Michigan 48824-1027

E-mail address: bao@math.msu.edu

Department of Mathematics, Michigan State University, East Lansing, Michigan 48824-1027

E-mail address: zhangh20@msu.edu

Department of Mathematics, The Chinese University of Hong Kong, Shatin, N.T., Hong Kong, People's Republic of China

E-mail address: zou@math.cuhk.edu.hk 